\title{
Research Article \\ Geometry Design of Coaxial Rigid Rotor in High-Speed Forward Flight
}

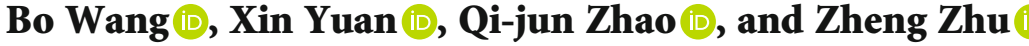 \\ National Key Laboratory of Rotorcraft Aeromechanics, Nanjing University of Aeronautics and Astronautics, Nanjing, \\ Jiangsu 210016, China \\ Correspondence should be addressed to Bo Wang; wangbo@nuaa.edu.cn
}

Received 13 October 2020; Revised 16 November 2020; Accepted 24 November 2020; Published 7 December 2020

Academic Editor: Jacopo Serafini

Copyright ( 2020 Bo Wang et al. This is an open access article distributed under the Creative Commons Attribution License, which permits unrestricted use, distribution, and reproduction in any medium, provided the original work is properly cited.

\begin{abstract}
The aerodynamic performance analysis and blade planform design of a coaxial rigid rotor in forward flight were carried out utilizing CFD solver CLORNS. Firstly, the forward flow field characteristics of the coaxial rotor were analyzed. Shock-induced separation occurs at the advancing side blade tip and severe reverse flow occurs at the retreating side blade root. Then, the influence of geometrical parameters of the coaxial rigid rotor on forward performance was investigated. Results show that swept-back tip could reduce the advancing side compressibility drag and elliptic shape of blade planform could optimize the airload distribution at high advance ratio flights. A kind of blade planform combining swept-back tapered tip and nonlinear chord distribution was optimized to improve the rotor efficiency for a given high-speed level flight based on geometric parameter studies. The optimized coaxial rotor increases lift-to-drag ratio by $30 \%$ under the design conditions.
\end{abstract}

\section{Introduction}

Coaxial rigid rotor helicopters with auxiliary propulsion are able to attain better performance at high forward speed compared with conventional single-rotor helicopters. The application of lift offset on coaxial rigid rotors plays an important role in the improvement of forward rotor efficiency. For the single-rotor configuration, the forward flight speed is restricted by dynamic stall on the retreating side and compressibility on the advancing side of the disk. Lift capability of advancing blades is also limited to maintain lateral equilibrium. Lift offset concept of coaxial rotor offers a solution to the speed limitation problem. It allows the lateral asymmetry of the lift distribution. The advancing blades generate most lift of the disk, and the retreating blades are offloaded. The maintenance of lateral and directional equilibrium is achieved by balancing rolling moments and torque produced by the upper and lower rotors. However, the counterrotation of two rotors generates severe aerodynamic interaction, and operations are much more complicated. Here are limited analytical studies on coaxial rotor performance compared with single rotor configuration, as shown in the following.

Coleman [1] summarizes experimental and theoretical research on coaxial rotor aerodynamics up to 1997, among which hover measurement data from Harrington [2] and forward measurement data from Dingeldein [3] are commonly used for analysis validation. Barbely et al. [4] provided a comprehensive summary of computational investigations of coaxial rotors in hover and forward flight. Bagai and Leishman [5] developed a free vortex wake methodology (FVM) for multirotor configurations including coaxial rotors and investigated the wake structure of coaxial rotors. Brown and Line [6, 7] developed and extended Vorticity Transport Model (VTM) which performs well in retaining the structure and forming of the rotor wake. Kim and Brown $[8,9]$ analyzed the performance of coaxial rotors in steady and manoeuvring flight using VTM. Leishman and Ananthan [10, 11] established simple momentum theory and blade element momentum theory (BEMT) for coaxial rotor system in hover and axial flight. The viscous vortex particle method (VVPM) [12] is also applied into coaxial rotor studies. Tan et al. [13] 
and Singh et al. [14] used VVPM with vortex panel loads for coaxial rotor simulations. Computational fluid dynamics (CFD) is increasingly powerful with the development of numerical simulation techniques and computer hardware. CFD could provide an accurate prediction on performance. Rajmohan et al. [15] developed a hybrid VPM/CFD methodology to study coaxial rotor aerodynamics, which maintains high accuracy and efficiency. CFD and hybrid-CFD flow solvers such as OVERFLOW, Helios, and RotCFD are used to study the performance and flow physics of coaxial rotors $[4,16-18]$, and performance predictions are in good agreement with the experimental data.

There are more considerations in blade geometry design compared with conventional single rotor. Efforts on coaxial rotor design are made based on various above-mentioned methodologies. Leishman and Ananthan [11] gave the optimum blade twist of hovering Harrington coaxial rotor through BEMT method. Johnson et al. [19] designed a compound helicopter utilizing lift offset rotors using CAMRAD II, and the tapering planform and blade twist were optimized under the design conditions. Yeo and Johnson [20] carried out rotor planform and twist optimization for hover and cruise performance of high blade loading coaxial rotors by CAMRAD II. Bagai [21] described the aerodynamic design of $\mathrm{X} 2^{\mathrm{TD}}$ main rotor in detail including blade planform, twist distribution and airfoil configurations, and performance improvements it contributes to.

In previous work, a coaxial rotor solver has been developed based on CLORNS [22], a high-fidelity CFD solver. Hover performance of coaxial rotor has been investigated using the established CFD tool [23] by the present authors. These studies have proved that our CFD solver could provide a reliable performance prediction of coaxial rotor. An optimization method combining the surrogate-based approach and genetic algorithm was implemented in the blade geometry shape design $[24,25]$. In the present paper, the study of impacts of different geometry parameters on forward aerodynamic performance is added to explain why a rotor planform combining elliptic shape and swept-back tapered tip is chosen. The design operating condition was level cruise at 210 knots. At high advance ratio flights, compressibility on the advancing blade tip is one of the main causes of rotor performance deficiency. The geometry combination of elliptic shape and swept-back tapered tip could improve the rotor efficiency by providing a better airload distribution over the disk. The optimization was carried out based on the baseline geometry, and the optimum blade has a higher lift-to-drag ratio.

\section{Performance Prediction of Coaxial Rotors in Forward Flight}

2.1. Methodology Descriptions. Rotor performance is predicted using a coaxial rotor solver based on CLORNS [22]. The governing equations for rotor are the 3D compressible Reynolds Navier-Stokes equations (RANS) and for background are the Euler equations. Finite volume method (FVM) is used for spatial discretization. The governing equations for rotor flowfield are as follows:
TABLE 1: Computation method.

\begin{tabular}{lcc}
\hline & Background & Blade \\
\hline Governing equations & Euler equations & Compressible RANS \\
Spatial discretization & Cell-centered FVM \\
Time integration & LU-SGS \\
Inviscid flux & Roe-MUSCL \\
Viscous flux & 2nd-order central difference method \\
Turbulence model & SA model \\
\hline
\end{tabular}

$$
\frac{\partial}{\partial t} \iiint_{\Omega} \mathbf{W} d \Omega+\iint_{\partial \Omega}\left(\mathbf{F}_{\mathrm{c}}-\mathbf{F}_{\mathrm{v}}\right) d S=0
$$

where $\Omega$ is the control volume and $\partial \Omega$ is its closed surface; $d S$ is the surface element, $\mathbf{W}$ is the vector of conservative variables, $\mathbf{F}_{c}$ is the vector of convective fluxes, and $\mathbf{F}_{\mathrm{v}}$ is the vector of viscous fluxes.

$$
\begin{aligned}
\mathbf{W}=\left[\begin{array}{c}
\rho \\
\rho u \\
\rho v \\
\rho w \\
\rho E
\end{array}\right], \\
\mathbf{F}_{\mathrm{c}}=\left[\begin{array}{c}
\rho v V_{r}+n_{y} p \\
\rho w V_{r}+n_{z} p \\
\rho u V_{r}+n_{x} p \\
\rho H V_{r}+V_{m} p
\end{array}\right], \\
0 \\
\mathbf{F}_{\mathrm{v}}=\left[\begin{array}{c}
0 \\
n_{x} \tau_{x x}+n_{y} \tau_{x y}+n_{z} \tau_{x z} \\
n_{x} \tau_{y x}+n_{y} \tau_{y y}+n_{z} \tau_{y z} \\
n_{x} \tau_{z x}+n_{y} \tau_{z y}+n_{z} \tau_{z z} \\
n_{x} \Theta_{x}+n_{y} \Theta_{y}+n_{z} \Theta_{z}
\end{array}\right],
\end{aligned}
$$

where $V_{r}=\left(\mathbf{V}-\mathbf{V}_{b}\right) \cdot \mathbf{n}$ and $V_{m}=\mathbf{V}_{b} \cdot \mathbf{n}$, in which $\mathbf{V}_{b}=($ $\left.u_{b}, v_{b}, w_{b}\right)$ is the velocity of the moving blade grid, $\mathbf{V}=($ $u, v, w)$ is the velocity of the flow, and $\mathbf{n}=\left(n_{x}, n_{y}, n_{z}\right)$ is the normal vector to the surface pointing outward from the control volume. $\rho, p, E$, and $H$ represent density, pressure, the total energy, and the total enthalpy, respectively. $\tau_{(\cdot)}$ and $\Theta_{(\cdot)}$ represent the viscous stress and the work performed by the viscous stress and heat conduction, respectively. 


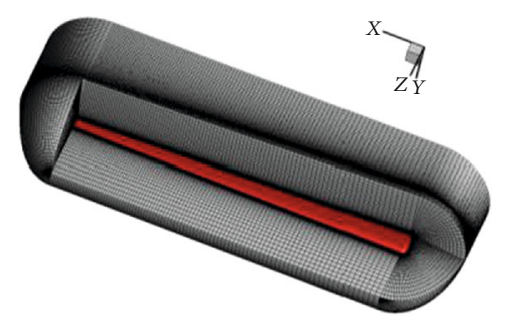

(a) Structured blade grid

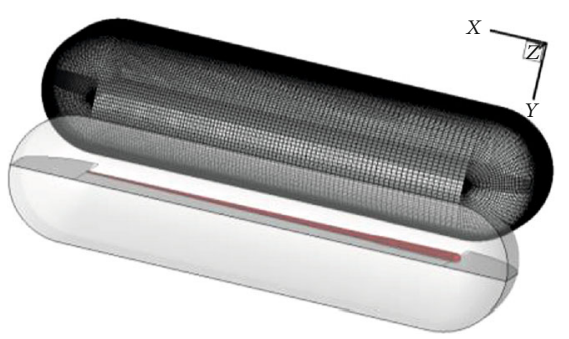

(b) Dual rotor grids of blade crossing

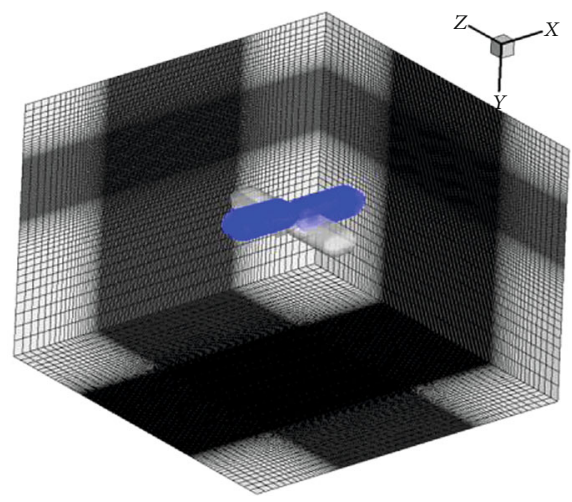

(c) Moving-embedded grid system

Figure 1: Computational grid for coaxial rotor.

TABLE 2: Convergence of grids.

\begin{tabular}{lcccc}
\hline & Background & Blade & Total/10 & Error of thrust coefficient \\
\hline Coarse & $175 \times 135 \times 159$ & $179 \times 39 \times 61$ & 5.46 & $4.73 \%$ \\
Medium & $201 \times 155 \times 187$ & $199 \times 51 \times 91$ & 9.52 & $2.61 \%$ \\
Fine 1 & $241 \times 201 \times 231$ & $221 \times 89 \times 101$ & 19.14 & $0.44 \%$ \\
Fine 2 & $361 \times 243 \times 351$ & $241 \times 101 \times 159$ & 46.27 & Benchmark \\
\hline
\end{tabular}

$$
\begin{aligned}
& \begin{cases}\tau_{x x}=2 \mu \frac{\partial u}{\partial x}-\left(\frac{2}{3}\right) \mu \nabla \cdot \mathbf{V}, & \tau_{x y}=\mu\left(\frac{\partial u}{\partial y}+\frac{\partial v}{\partial x}\right) \\
\tau_{y y}=2 \mu \frac{\partial v}{\partial y}-\left(\frac{2}{3}\right) \mu \nabla \cdot \mathbf{V}, & \tau_{y z}=\mu\left(\frac{\partial v}{\partial z}+\frac{\partial w}{\partial y}\right), \\
\tau_{z z}=2 \mu \frac{\partial w}{\partial z}-\left(\frac{2}{3}\right) \mu \nabla \cdot \mathbf{V}, & \tau_{x z}=\mu\left(\frac{\partial u}{\partial z}+\frac{\partial w}{\partial x}\right),\end{cases} \\
& \left\{\begin{array}{l}
\Theta_{x}=u \tau_{x x}+v \tau_{x y}+w \tau_{x z}+\kappa \frac{\partial T}{\partial x} \\
\Theta_{y}=u \tau_{y x}+v \tau_{y y}+w \tau_{y z}+\kappa \frac{\partial T}{\partial y} \\
\Theta_{z}=u \tau_{z x}+v \tau_{z y}+w \tau_{z z}+\kappa \frac{\partial T}{\partial z}
\end{array}\right.
\end{aligned}
$$

where $\mu$ denotes the dynamic viscosity coefficient, $\kappa$ is the thermal conductivity coefficient, and $T$ is the absolute static temperature.

Time integration is performed using the Lower-Upper Symmetric Gauss-Siedel (LU-SGS) method. The chosen time-steps correspond to $1.0^{\circ}$ of azimuth. The inviscid spatial terms are computed using a third-order MUSCL scheme with Roe's flux difference splitting, and the viscous terms are computed using second-order central differencing. The SpalartAllmaras turbulence model is employed for the RANS closure. Calculation methods are summarized in Table 1.

To allow for the counterrotation between the upper rotor and lower rotor, moving-embedded grid system is used. Figure 1 shows the structured grid of moving-embedded grid system. Figure 1(a) is the structured blade grid. Compared with the single rotor, the outer boundary of the blade grid should be set carefully to avoid intersections with another rotor blade. Figure 1(b) shows the relative position of the upper and lower blades when blade crossing occurs. It can be seen that there is some spacing between the outer boundary of upper blade grid and the lower blade surface; the same is between the outer boundary of lower blade grid and upper blade surface. Therefore, the transmission of flowfield information between blade grids and background grid at the end of each subiteration could work correctly.

The blade collective, lateral cyclic, and longitudinal cyclic pitch angles for each rotor under specific conditions are determined using the Newton-Raphson method. By 
trimming solution, the upper and lower rotors are torque balanced and achieve the target thrust coefficient, rolling, and pitching moments. The blade pitch $\theta$ of each rotor at azimuth $\psi$ is as follows:

$$
\begin{gathered}
\theta_{U}=\theta_{0 U}+\theta_{1 c U} \cos \psi_{U}+\theta_{1 s U} \cos \psi_{U} \\
\theta_{L}=\theta_{0 L}+\theta_{1 c L} \cos \psi_{L}+\theta_{1 s L} \cos \psi_{L}
\end{gathered}
$$

In the trimming procedure, the rotor control input vector and the response vector are, respectively, given by

$$
\begin{gathered}
X=\left\{\theta_{0 U}, \theta_{1 s U}, \theta_{1 c U}, \theta_{0 L}, \theta_{1 s L}, \theta_{1 c L}\right\}, \\
Y=\left\{\sum C_{T}, \sum C_{Q}, \text { LOS }, C_{M z U}, \sum C_{M x}, C_{M z L}\right\},
\end{gathered}
$$

where $C_{T}, C_{Q}$, LOS, $C_{M z}$, and $C_{M x}$ represent the thrust coefficient, torque coefficient, lift offset, pitching moment coefficient, and rolling moment coefficient, respectively. Subscripts $U$ and $L$ represent the upper rotor and lower rotor, respectively. $\sum C_{T}=C_{T U}+C_{T L}, \quad \sum C_{Q}=C_{Q U}+C_{Q L}$, $\sum C_{M x}=C_{M x U}+C_{M x L}$, and LOS $=\left|C_{M x U}\right|+\left|C_{M x L}\right| / \sum C_{T} \cdot R$.

Then, the equation to solve is

$$
\begin{gathered}
Y(X)-Y^{\text {target }}=0 \\
X^{(n+1)}=X^{(n)}-J^{-1}\left(Y^{(n)}-Y^{\text {target }}\right),
\end{gathered}
$$

where $Y^{\text {target }}=\left\{\sum C_{T}{ }^{\text {target }}, 0, \operatorname{LOS}^{\text {target }}, 0,0,0\right\}$, and $J$ is the Jacobian matrix.

$$
J=\left[\begin{array}{cccccc}
\frac{\partial \sum C_{T}}{\partial \theta_{0 U}} & \frac{\partial \sum C_{T}}{\partial \theta_{1 s U}} & \frac{\partial \sum C_{T}}{\partial \theta_{1 c U}} & \frac{\partial \sum C_{T}}{\partial \theta_{0 L}} & \frac{\partial \sum C_{T}}{\partial \theta_{1 s L}} & \frac{\partial \sum C_{T}}{\partial \theta_{1 c L}} \\
\frac{\partial \sum C_{Q}}{\partial \theta_{0 U}} & \frac{\partial \sum C_{Q}}{\partial \theta_{1 s U}} & \frac{\partial \sum C_{Q}}{\partial \theta_{1 c U}} & \frac{\partial \sum C_{Q}}{\partial \theta_{0 L}} & \frac{\partial \sum C_{Q}}{\partial \theta_{1 s L}} & \frac{\partial \sum C_{Q}}{\partial \theta_{1 c L}} \\
\frac{\partial \mathrm{LOS}}{\partial \theta_{0 U}} & \frac{\partial \mathrm{LOS}}{\partial \theta_{1 s U}} & \frac{\partial \mathrm{LOS}}{\partial \theta_{1 c U}} & \frac{\partial \mathrm{LOS}}{\partial \theta_{0 L}} & \frac{\partial \mathrm{LOS}}{\partial \theta_{1 s L}} & \frac{\partial \mathrm{LOS}}{\partial \theta_{1 c L}} \\
\frac{\partial C_{M z U}}{\partial \theta_{0 U}} & \frac{\partial C_{M z U}}{\partial \theta_{1 s U}} & \frac{\partial C_{M z U}}{\partial \theta_{1 c U}} & \frac{\partial C_{M z U}}{\partial \theta_{0 L}} & \frac{\partial C_{M z U}}{\partial \theta_{1 s L}} & \frac{\partial C_{M z U}}{\partial \theta_{1 c L}} \\
\frac{\partial \sum C_{M x}}{\partial \theta_{0 U}} & \frac{\partial \sum C_{M x}}{\partial \theta_{1 s U}} & \frac{\partial \sum C_{M x}}{\partial \theta_{1 c U}} & \frac{\partial \sum C_{M x}}{\partial \theta_{0 L}} & \frac{\partial \sum C_{M x}}{\partial \theta_{1 s L}} & \frac{\partial \sum C_{M x}}{\partial \theta_{1 c L}} \\
\frac{\partial C_{M z L}}{\partial \theta_{0 U}} & \frac{\partial C_{M z L}}{\partial \theta_{1 s U}} & \frac{\partial C_{M z L}}{\partial \theta_{1 c U}} & \frac{\partial C_{M z L}}{\partial \theta_{0 L}} & \frac{\partial C_{M z L}}{\partial \theta_{1 s L}} & \frac{\partial C_{M z L}}{\partial \theta_{1 c L}}
\end{array}\right] .
$$

2.2. Validations for Coaxial Rotor. The experimental data of Harrington Rotor-1 [2,3] are used to validate the computational predictions. It consists of one 2-bladed rotors arranged to form a single system (HS1) and the same two 2-bladed rotors arranged to form a coaxial system (HC1). The blade uses a NACA airfoil with a nonlinearly varying thickness and a linearly varying chord length. The tip velocity is $142.95 \mathrm{~m} / \mathrm{s}$ in forward flight.

Four groups of moving-embedded grids are generated to investigate the mesh convergence. Fine 2 gird is the finest, whose thrust coefficient is chosen as benchmark. The grid

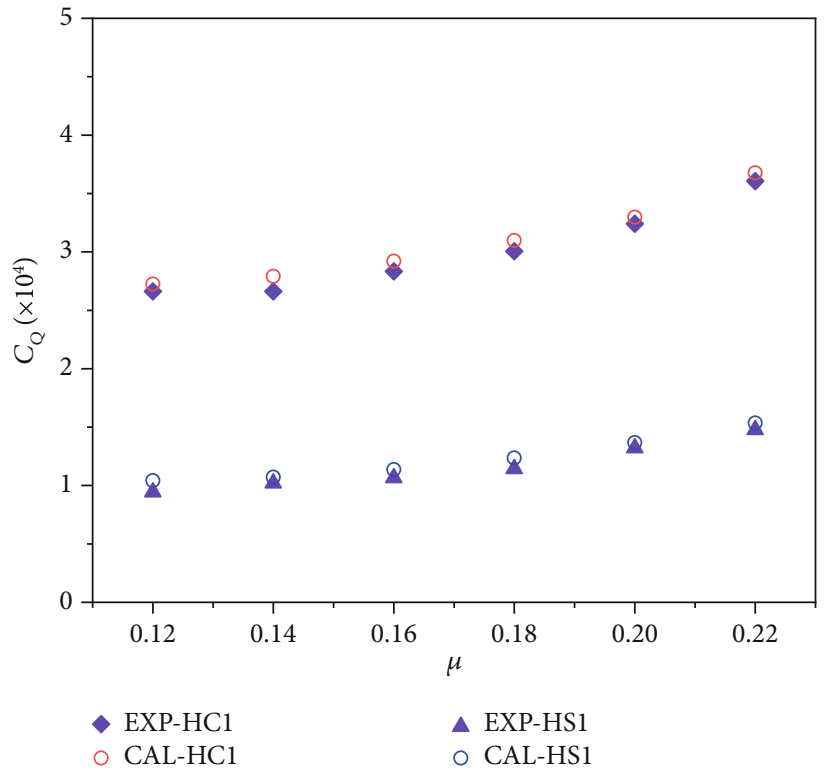

FIGURE 2: CFD calculations compared with measured performance of $\mathrm{HC} 1$ and $\mathrm{HS} 1$ in forward flight.

number and its thrust coefficient comparisons are shown in Table 2. Fine 1 grid was finally chosen into calculation. The size of Fine 1 background grid surrounding the rotor gird is refined to $0.05 c$ ( $c$ is the blade tip chord length), under which size the mesh convergence is achieved. The subiteration of the solver is set as 50 , and then, the residual of density will be reduced by at least 1 order.

Figure 2 shows the measured forward flight performance of $\mathrm{HC} 1$ and $\mathrm{HS} 1$ compared to calculations. The calculations are in agreement with the measurements. The established CFD solver overpredicted the forward performance, and the calculations are closer to the experimental data with the increase of advance ratio. It also shows that coaxial rotor costs more power than twice of single-rotor consumptions, which could be mainly attributed to the severe interference in the flowfield of coaxial rotor caused by the counterrotation of upper rotor and lower rotor. Figure 3 gives the convergence of thrust coefficient of coaxial rotor at $\mu=0.2$. It shows that the force came to convergence after 2 revolutions under a given trimming operations.

\subsection{Aerodynamic Features of Coaxial Rigid Rotors in Forward} Flight. The model coaxial rotor used for analysis consists of two 2-bladed rotors with rectangular planform. The blades have a NACA0012 profile with a radius of $2.0 \mathrm{~m}$ and feature a linear aerodynamic twist rate of $-5 \mathrm{deg} / \mathrm{m}$. The rotor aspect ratio is 10 . The separation distance between the upper rotor and lower rotor is $0.15 \mathrm{R}$. The setting of initial azimuthal locations is shown in Figure 4: U1 (upper rotor blade 1) is at $90^{\circ}$, $\mathrm{U} 2$ (upper rotor blade 2) is at $270^{\circ}, \mathrm{L} 1$ (lower rotor blade 1 ) is at $0^{\circ}$, and L2 (lower rotor blade 2) is at $180^{\circ}$.

The tip mach speed is 0.528 for calculations in the present paper. All calculations are trimmed. The torque is balanced with a thrust coefficient of 0.013 and a lift offset of 0.35 . Rotor shaft angle is $0^{\circ}$. The equivalent lift to drag ratio of coaxial 


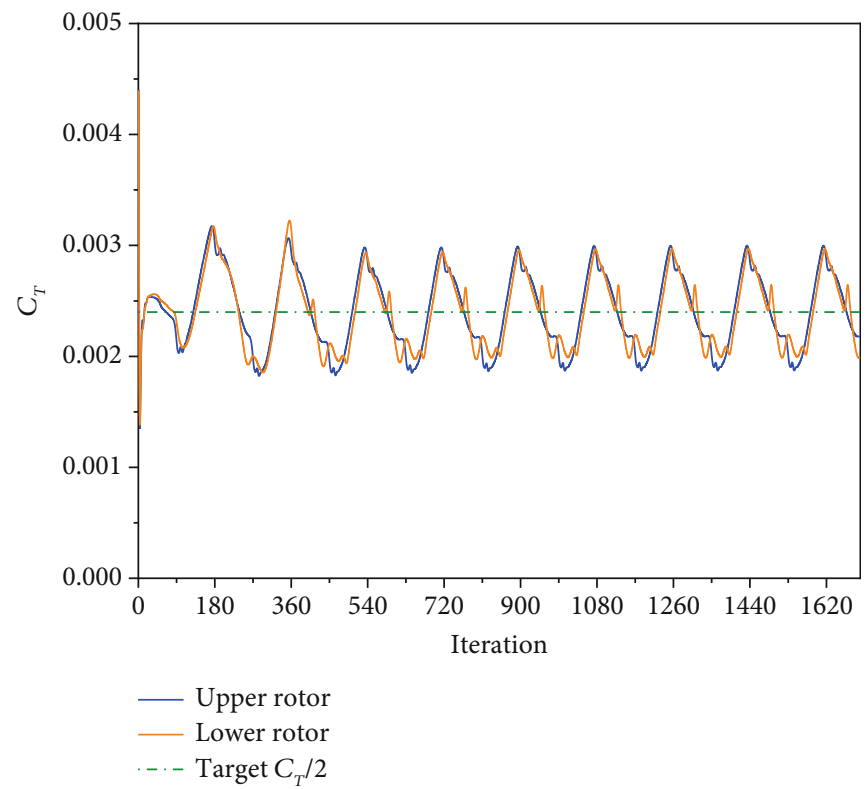

FIGURE 3: The convergence of thrust coefficients of the upper and lower rotors.

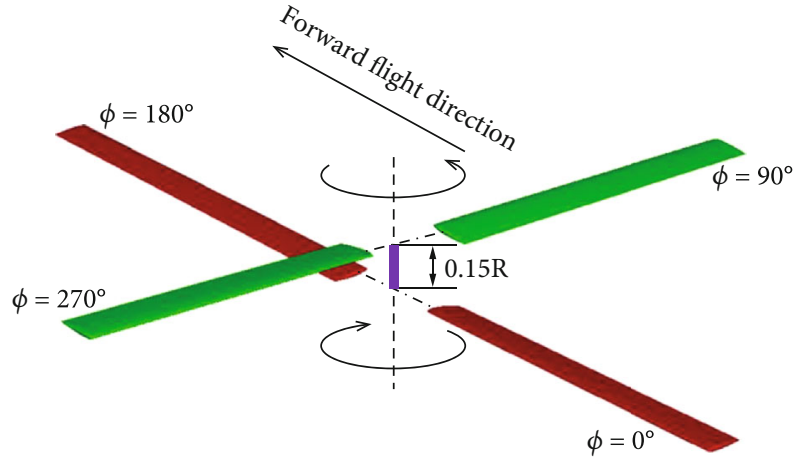

FIGURE 4: Blade azimuthal locations at the beginning.

rotor is defined as

$$
L / D=\frac{\sum C_{L}}{\sum C_{D}+\sum C_{Q} / \mu} .
$$

The blade mesh has $251 \times 131 \times 80$ points in the streamwise, spanwise, and normal directions, respectively, and the background mesh has $241 \times 281 \times 201$ points in the lateral, longitudinal, and vertical directions, respectively. Simulation case used for analysis is set at advance ratio of 0.6. To understand the performance characteristics of coaxial rotor in high-speed forward flight, the lift distribution over the rotor disk is given in Figure 5 in the form of $C_{L} \bullet \mathrm{Ma}^{2}$ contour. It shows that the lift is mainly generated by the advancing blades, and the retreating side provides almost nonlift. This loading distribution is a consequence of the application of the lift offset. The tip regions at $\psi=90^{\circ}$ are negatively loaded at this condition, and its area is smaller than the single-rotor configuration due to the application of lift offset.
Good insight into blade flow features is made by looking the upper surface streamlines during one revolution in Figure 6. In the current operating state, there are little differences between the flow topologies of the upper rotor and lower rotor. Therefore, here only gives the detailed flow topology on the upper surface of the upper rotor. It can be seen that the flow feature varies with the azimuth angle and the radical position. There is an obvious deviation of the flow over a large area (radius above $0.95 \mathrm{R}$ ) at $\psi=90^{\circ}$. Shock wave occurs in this area, which produces shock-induced separation. And reverse flow occurs in the root regions on the retreating side, generating large profile drag and negative lift. The area of reverse flow depends on the flight speed and rotor frequency. Radical flow is more significant on the retreating side. Figure 7 shows the temporal variation of CT over one revolution. From the figure, the unsteadiness is clearly seen with a dominant $2 / \mathrm{rev}$ frequency (blade number of one rotor).

For a coaxial rotor with two 2-bladed counterrotating rotors, there are 4 overlaps for each blade in one rotor revolution as shown in Figure 8. According to the research of Lakshminarayan and Baeder [18], blade overlaps generate significant impulses in the instantaneous thrust and power in hover, which could be explained by the blade thickness (a venturi effect) and loading (an upwash-downwash effect). Similarly, blade overlaps exist in forward flight and have effects on aerodynamic loading. The blades of the upper rotor overlap with blades of the lower rotor in the following order for one revolution: 1/8 Rev, 3/8 Rev, 5/8 Rev, and 7/8 Rev (Rev means one revolution). Figure 9 shows the pressure distribution of $0.95 \mathrm{R}$ blade section in $1 / 8 \mathrm{Rev}$ and $3 / 8 \mathrm{Rev}$ when the two sections begin to across.

It can be seen that sectional pressure distributions of $1 / 8 \mathrm{Rev}$ and $3 / 8 \mathrm{Rev}$ are different. This is because $\mathrm{U} 1 / \mathrm{L} 1$ is advancing blades at $1 / 8 \mathrm{Rev}$ time and $\mathrm{U} 2 / \mathrm{L} 1$ is advancing 


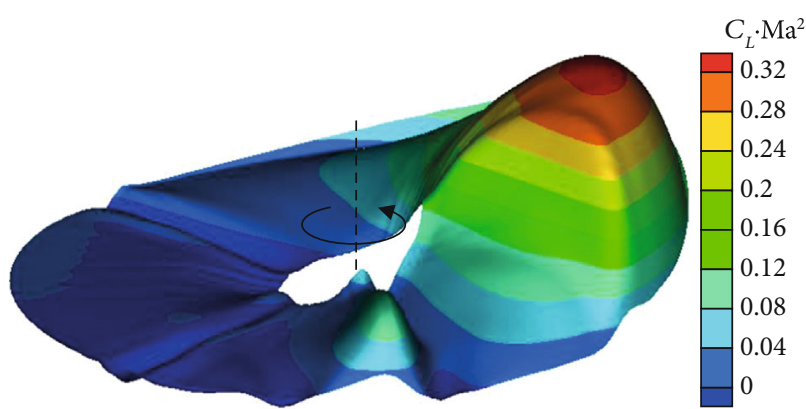

(a) Upper rotor

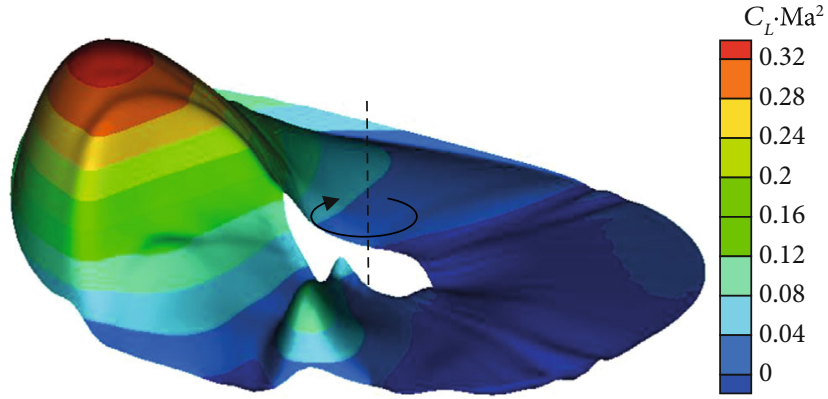

(b) Lower rotor

Figure 5: $C_{L} \bullet \mathrm{Ma}^{2}$ contours over the rotor disk.
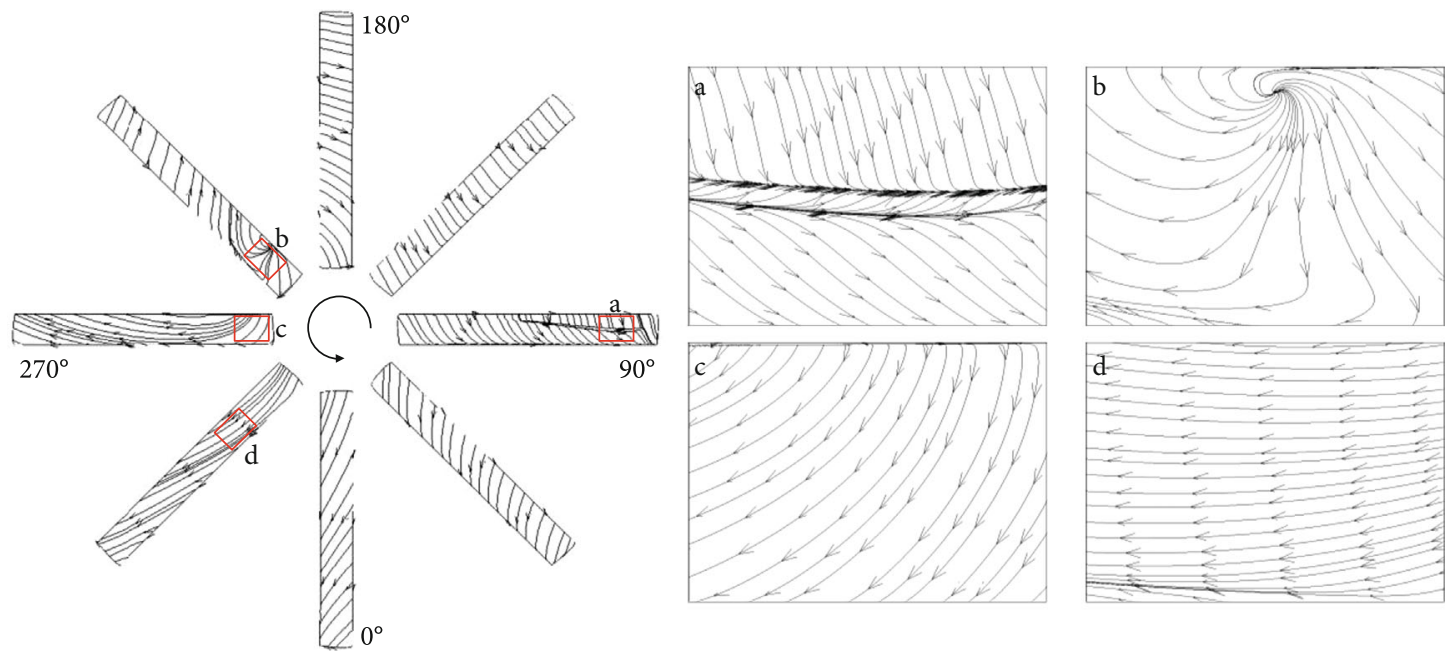

FIgURE 6: Streamlines on the upper surface of the upper rotor during one revolution.

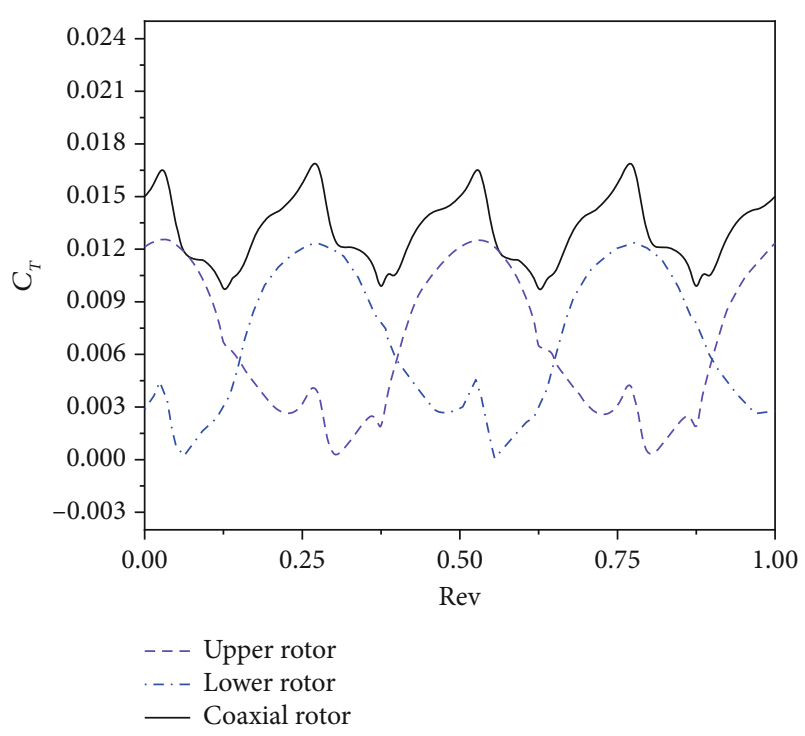

Figure 7: Temporal variation of $C_{T}$ of the upper and lower rotors over one revolution. blades at $3 / 8 \mathrm{Rev}$ time. The investigated case has a lift offset of 0.35 after trimming. Therefore, the blades are unloaded when they are on the retreating side through the adjustment of cyclic pitch. Figure 9 reveals a highly asymmetric pressure field below and above each airfoil section of the advancing blades, namely, lift is produced. And for the sections of retreating blades, U2/L2 in Figure 9(a)) and U1/L2 in Figure 9(b), the pressure field is close to symmetric, which means they produce little lift. In Figure 9(a), the airfoil section of L1 is affected by the presentation of U2 airfoil section, and in Figure 9(d), the airfoil section of L1 is affected by U1 airfoil section. It also shows that when the lower rotor blades are on the retreating side, there is only small interference between their flow fields. This phenomenon is mainly brought by the lift offset concept of $\mathrm{ABC}$ rotor, and the effect of the blade thickness is weaken compared with hover state.

The pressure contours on the upper surface of two rotors are shown in Figure 10, which represent typical positions during one revolution including crossing, and overlap. It shows that the low pressure area outside the blade appears when the upper rotor or lower rotor is on the advancing side. When on the retreating side, the blade surface has an even pressure distribution. 


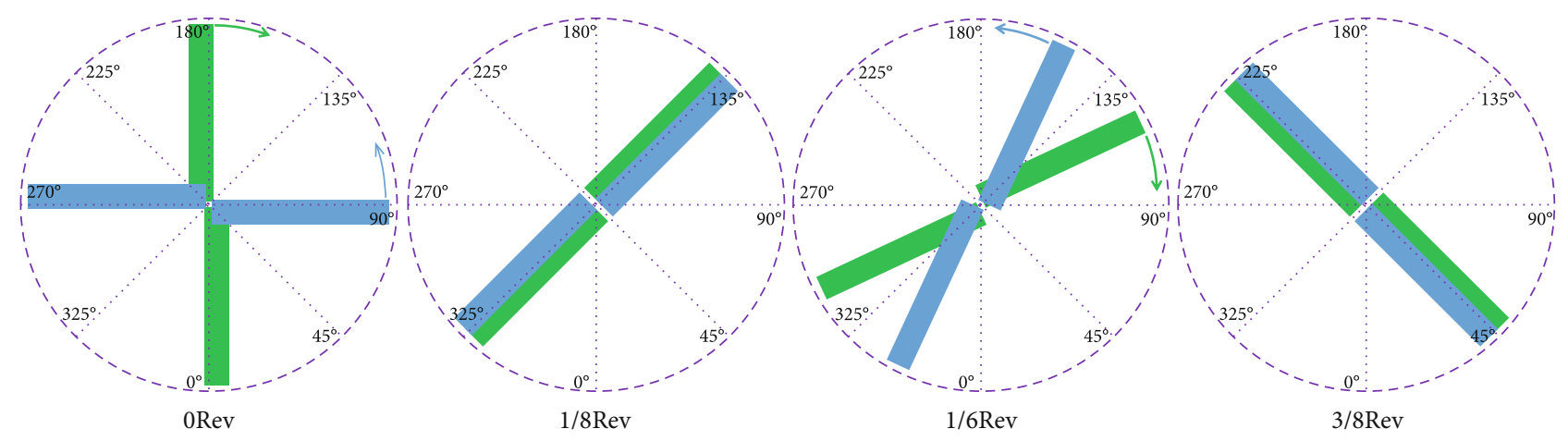

Upper rotor Lower rotor

FIgURE 8: Counterrotation of coaxial rotors.
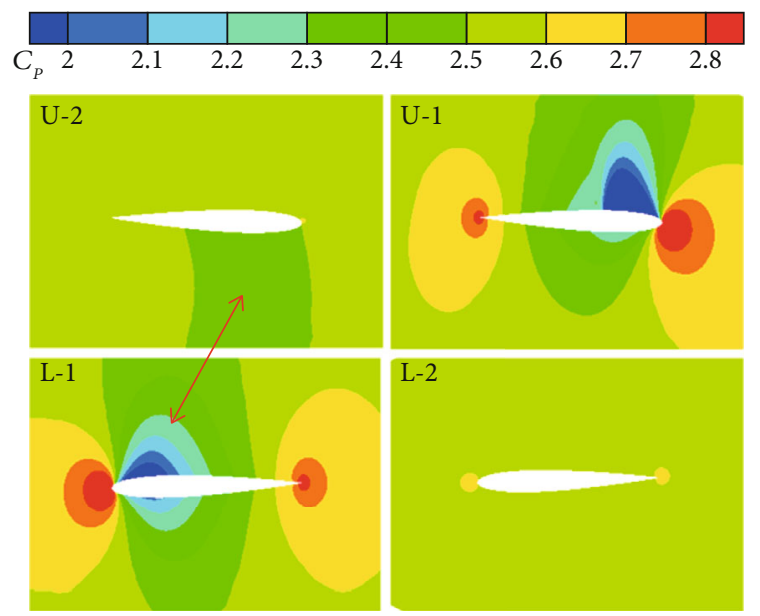

(a) $1 / 8 \operatorname{Rev}$
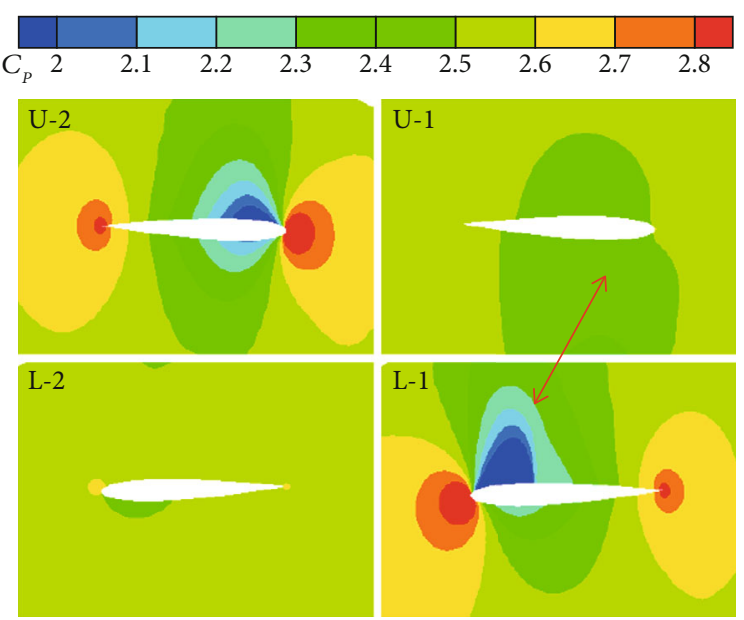

(b) $3 / 8 \mathrm{Rev}$

Figure 9: Nondimensional pressure distribution of blade section at $r / R=0.95$.

\section{Geometrical Parameter Influence Analysis}

\subsection{Swept-Back Tip}

3.1.1. Swept-Back Angle. Three types of blades with swept back tip are chosen to simulate the effect of swept-back angle on aerodynamic characteristics. All blades begin to sweep back at $0.9 \mathrm{R}$ radius, and the swept-back angles are $10^{\circ}, 20^{\circ}$, and $30^{\circ}$, respectively. Figure 11 shows the liftto-drag ratio of three blades compared with the rectangular blade (the rotor in Figure 4). In trimmed conditions, swept-back blades have better rotor efficiency than the rectangular blade, and this improvement increases with swept-back angle.

Case of $\mu=0.7$ is taken into analysis. Figure 12 shows the pressure distribution on the upper surface of blades with different swept-back angle at $90^{\circ}$ azimuthal position. There is an obvious area reduction of low pressure at 0.9R spanwise position of swept-back blades compared with the rectangular blade tip. And the low pressure center in the tip region moves outwards and is weakened with the increase of swept-back angle. The gain of swept-back tip is brought by the reduction in Mach number normal to the leading edge of blade tip sections, which is significant on the advancing side. Figure 13 shows the span thrust distribution at $90^{\circ}$ azimuthal position. Swept-back tips have higher thrust than the rectangular tip, and the thrust increases with swept-back angle. This is in conformity with the pressure distribution analysis in Figure 12. In the middle section, the thrust of blades with sweptback tip is lower than the rectangular blade.

Figure 14 shows the blade section pressure coefficient at $0.9 \mathrm{R}$, where the swept-back begins. It reveals that sweptback tips lower the negative pressure amplitude on the upper surface before the shock occurs (near 0.65c) in this position. With the increase of swept-back angle, the adverse pressure gradient is reduced. The shock wave moves toward the trailing edge, and its strength is weakened. Figure 15 shows the blade section pressure coefficient at $0.95 \mathrm{R}$. The 

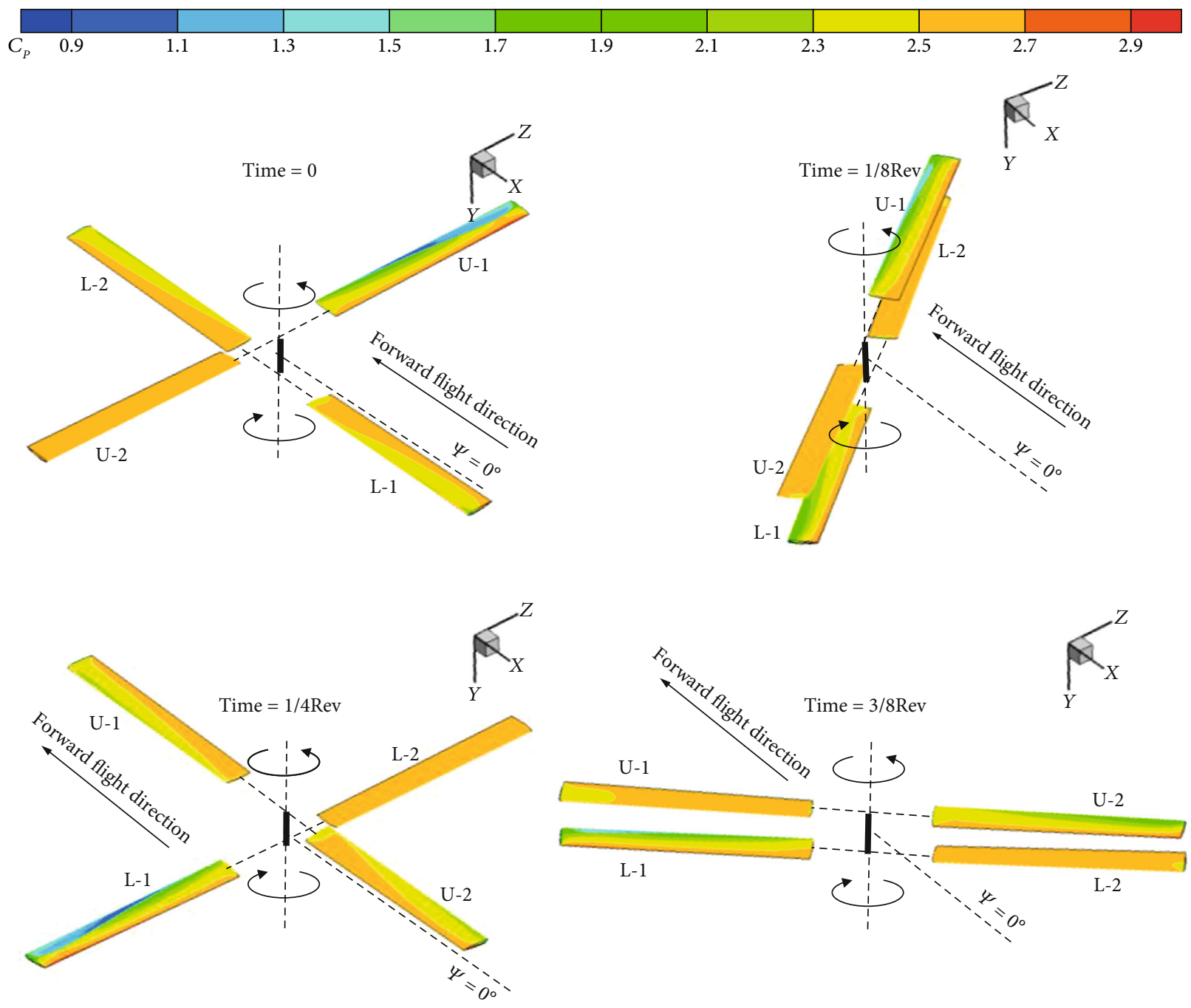

Figure 10: Pressure distribution on the upper surface of two rotors at 0 Rev, 1/8Rev, 1/4Rev, and 3/8Rev.

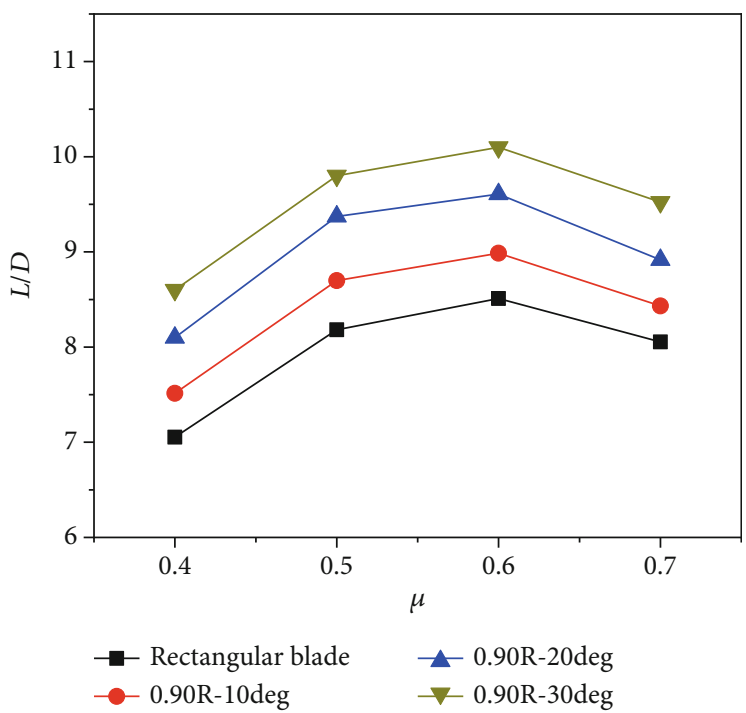

FIGURE 11: Lift-to-drag ratio of blades with different swept-back angle. upper surface pressure coefficients of three swept-back blades and the rectangular blade are nearly the same before the shock occurs. On the upper surface, air compressibility makes the position of pressure jump moves toward the leading edge. On the lower surface, the pressure jump decreases with the swept-back angle, and the shock strength is weakened.

3.1.2. Swept-Back Position. Three types of blades with swept back tip are chosen to simulate the effect of swept-back position on aerodynamic characteristics. All blades have a sweptback angle of $20^{\circ}$, and the swept-back positions begin at $0.85 \mathrm{R}, 0.9 \mathrm{R}$, and $0.95 \mathrm{R}$, respectively. Figure 16 shows the lift-to-drag ratio of three rotors compared with the rectangular blade (the rotor in Figure 4). In trimmed conditions, swept-back blades have better rotor efficiency than the rectangular blade, and this improvement increases with sweptback area.

Case of $\mu=0.7$ is taken into analysis. Figure 17 shows the pressure distribution on the upper surface of blades with different swept-back area at $90^{\circ}$ azimuthal position. Low 

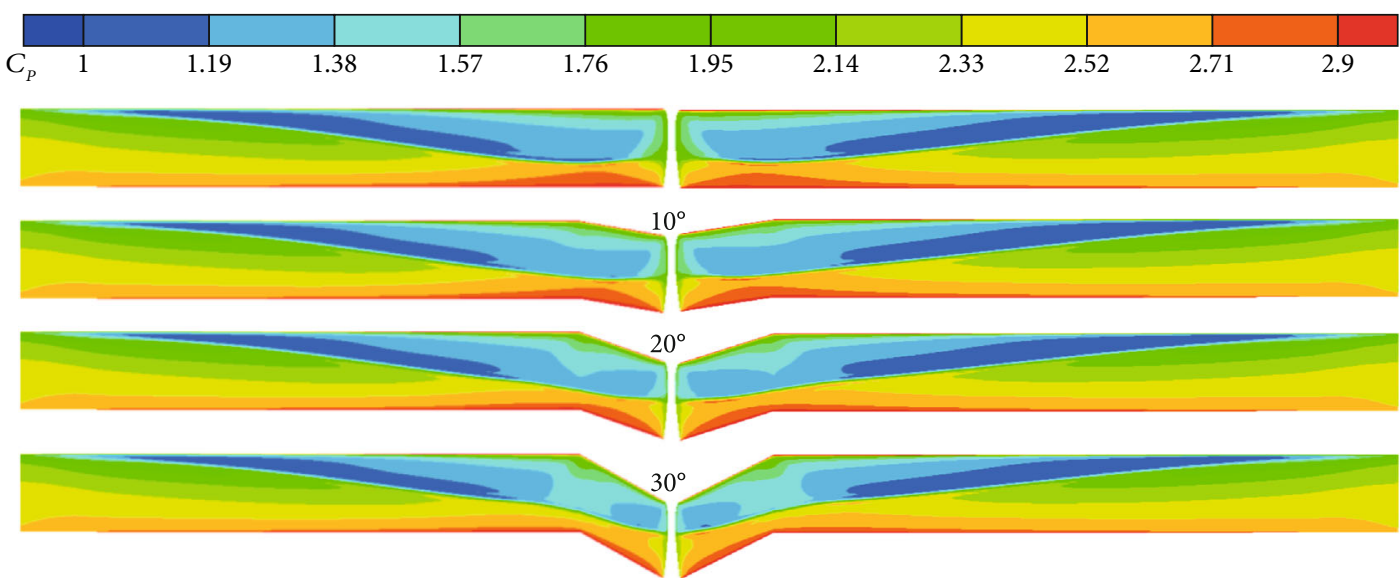

FIGURE 12: Pressure distribution on the upper surface of the upper rotor $\left(\psi=90^{\circ}, \mu=0.7\right)$.

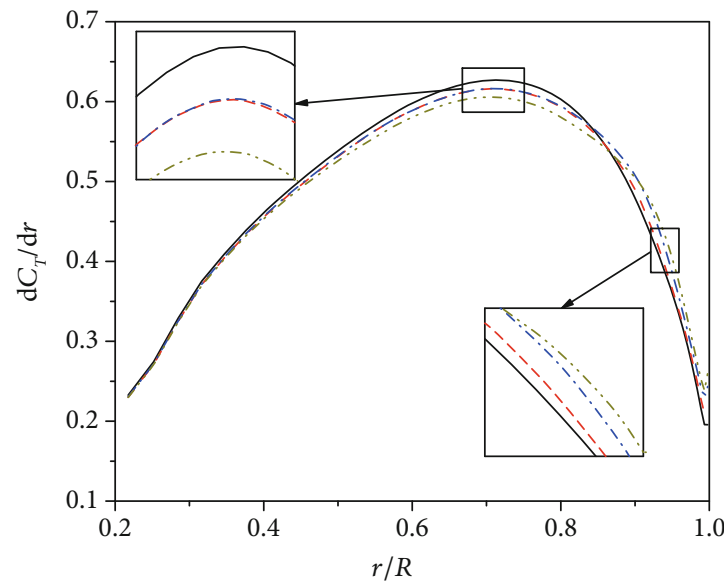

$\begin{array}{lll}\text { - Rectangular blade } \quad-.--0.90 \mathrm{R}-20 \mathrm{deg} \\ ---0.90 \mathrm{R}-10 \mathrm{deg} & -. .-0.90 \mathrm{R}-30 \mathrm{deg}\end{array}$

(a) Upper rotor

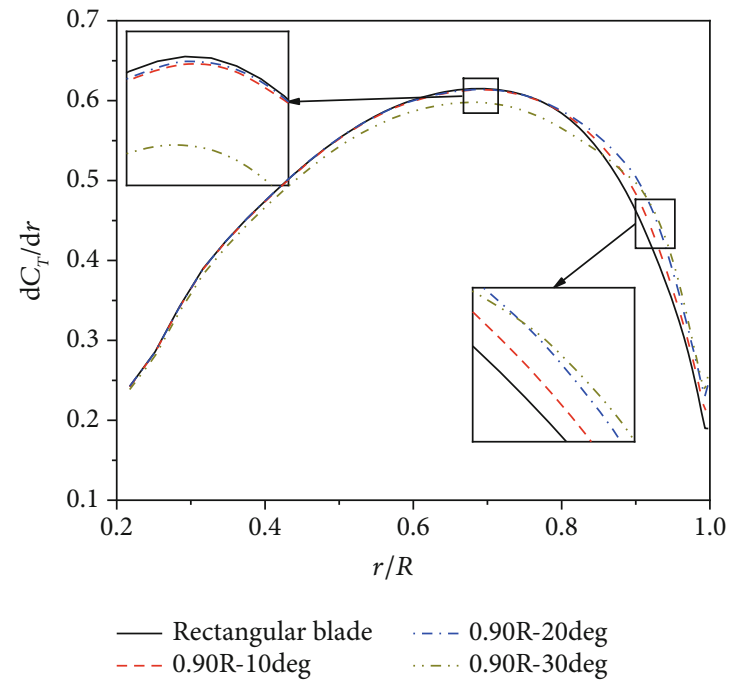

(b) Lower rotor

Figure 13: Span thrust distribution $\left(\psi=90^{\circ}, \mu=0.7\right)$.

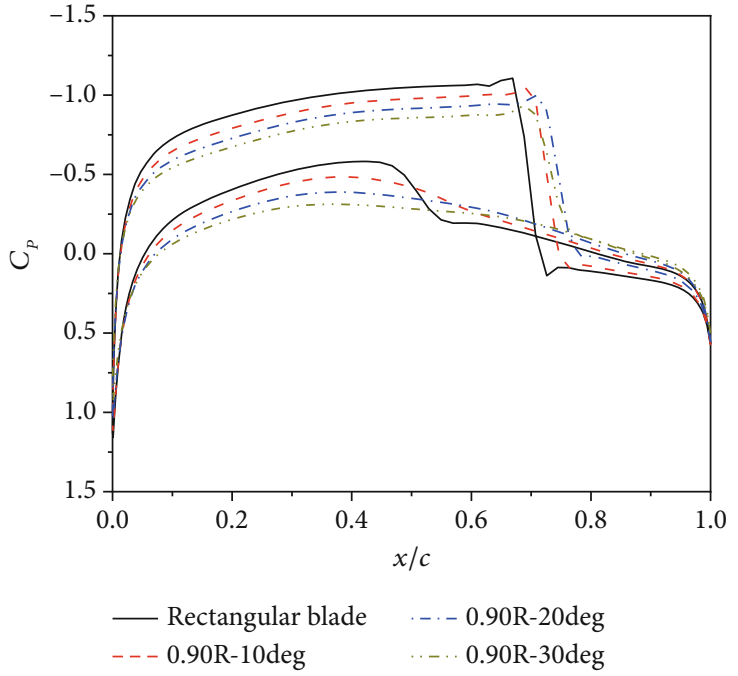

(a) Upper rotor

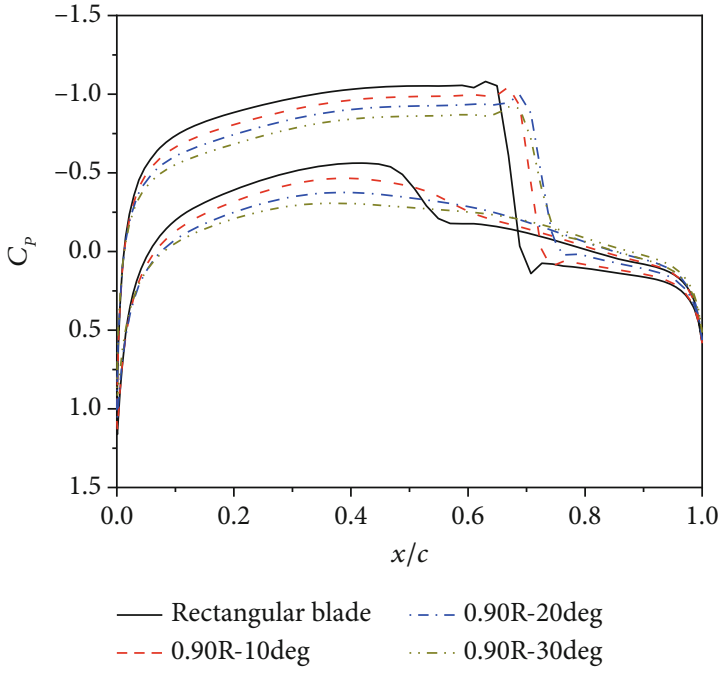

(b) Lower rotor

FIGURE 14: Blade section pressure coefficient $\left(\psi=90^{\circ}, \mu=0.7, r / R=0.90\right)$. 


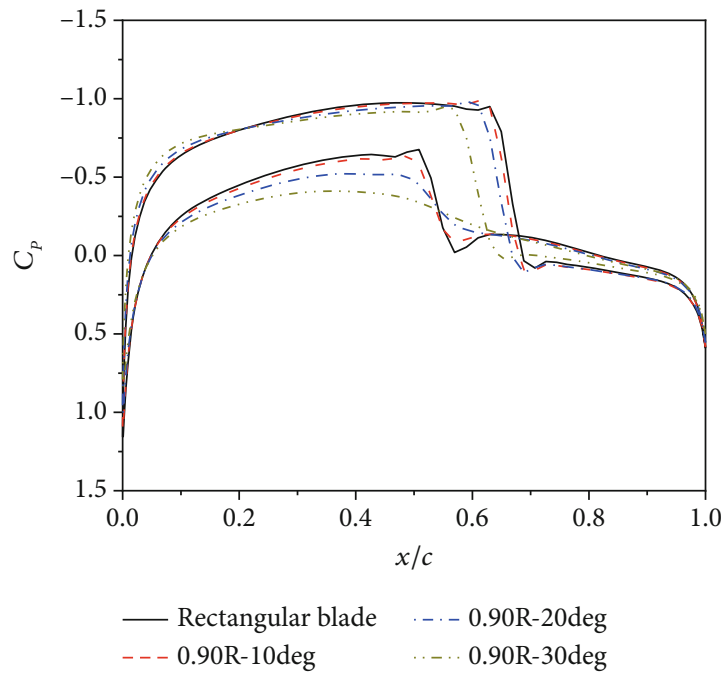

(a) Upper rotor

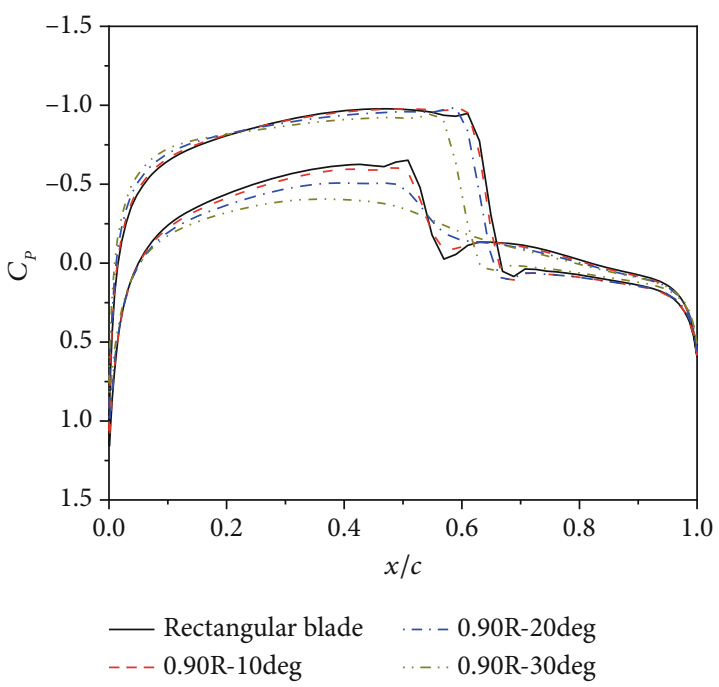

(b) Lower rotor

FIGURE 15: Blade section pressure coefficient $\left(\psi=90^{\circ}, \mu=0.7, r / R=0.95\right)$.

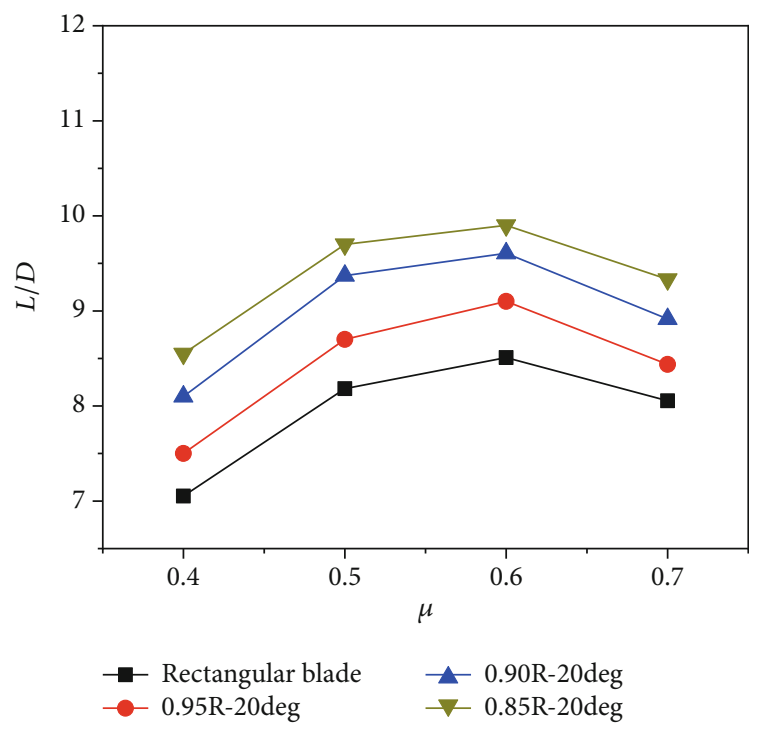

FIGURE 16: Lift-to-drag ratio of rotors with different swept-back position.

pressure region in the midblade (deep blue band in Figure 17) is narrowed with the increase of swept-back area. The swept-back tip reduces the shock wave drag, so the rotor efficiency is improved as shown in Figure 16. Figure 18 shows the spanwise thrust distribution at $90^{\circ}$ azimuthal position. Swept-back tips have lower thrust than the rectangular tip in the middle blade, and the thrust in the middle blade decreases with swept-back angle.

3.2. Nonlinear Chord Distribution. Three types of blades with elliptic shape resembling $\mathrm{X} 2^{\mathrm{TD}}$ rotor [5] are chosen to simulate the effects of chord distribution. The main part of the planform is an ellipse with specific length and position of the minor axis. The elliptic sections of three blades are cen- tered at $0.7 \mathrm{R}, 0.7 \mathrm{R}$, and $0.6 \mathrm{R}$. The length of the minor axes are $1.2 c, 1.35 c$, and $1.25 c$ ( $c$ is the length of the root chord), respectively. Figure 19 shows the lift-to-drag ratio variation of three rotors compared with the rectangular blade (the rotor in Figure 4). It reveals that the elliptic chord distribution has better forward performance than the rectangular planform. Among three elliptic blades, the blade " $0.6 R$ $1.35 c$ " has the highest $L / D$. Considering the higher $L / D$ of the blade " $0.7 R-1.35 c$ " compared with the blade " $0.7 R-1.20 c$ " may be brought by the change of rotor solidity, it implies that the rotor efficiency of elliptic blades is sensitive to the position of minor axes.

Case of $\mu=0.7$ is taken into analysis. Figure 20 shows the pressure distribution on the upper surface of blades with different chord distribution at $90^{\circ}$ azimuthal position. Figure 21 shows the spanwise thrust distribution at $90^{\circ}$ azimuthal position. There are obvious differences in the pressure distributions between the rectangular blade and elliptic blades. The strength of low pressure area of elliptic blades is weakened. This phenomenon may be brought by the leading edge profile, which is gently swept forward to the center of the ellipse and then gently swept back with the chord length tapering in the blade tip. Elliptic blade generates more lift in the middle part compared with rectangular blade as shown in Figure 21. The position of the lift peak moves towards the blade root with the center of the ellipse moving inwards. And the lift peak value increases with the length of the minor axis (the largest chord length of the blade). When performed in high advance ratio conditions, the blade tip of coaxial rotor needs to be unloaded. Therefore, the elliptic shape is in favor of high forward rotor efficiency through concentrating more lift in the middle part of blade.

Figure 22 shows the streamline on the upper surface of the upper rotor. In the current state $(\mu=0.7)$, severe shockinduced separation occurs on the blade surface on the 


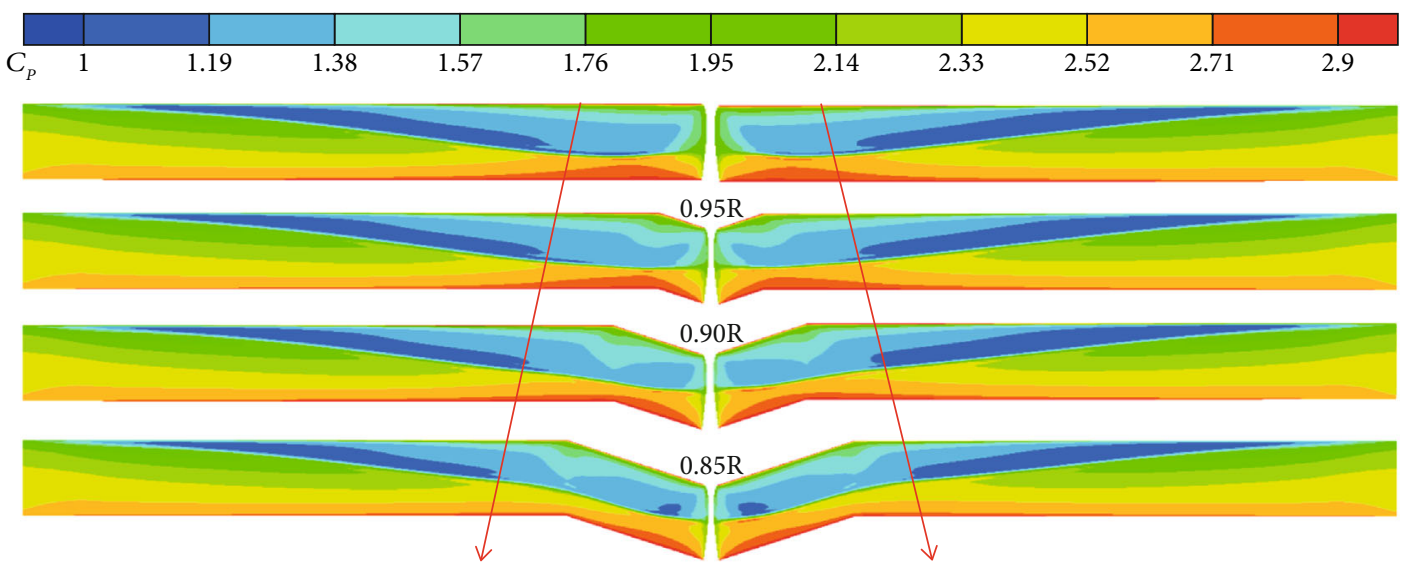

Figure 17: Pressure distribution on the upper surface of the upper rotor $\left(\psi=90^{\circ}, \mu=0.7\right)$.

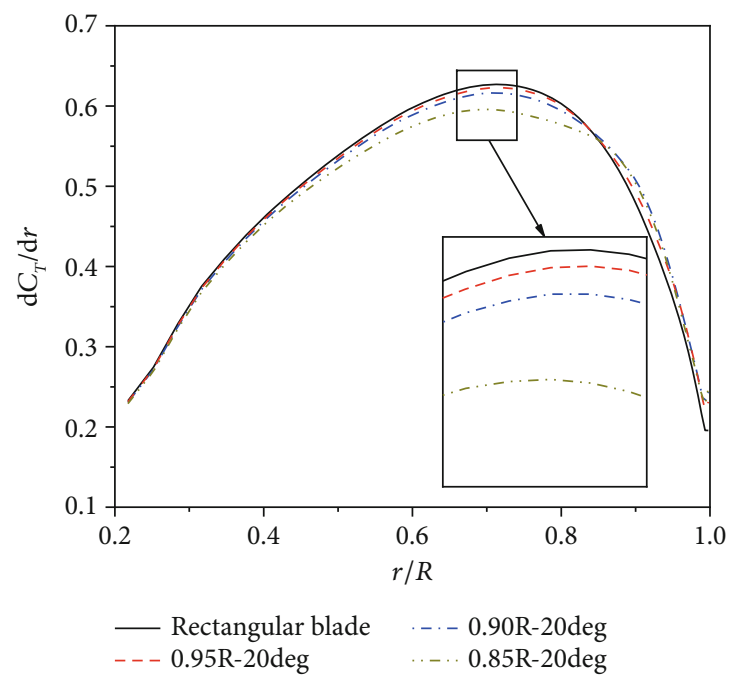

(a) Upper rotor

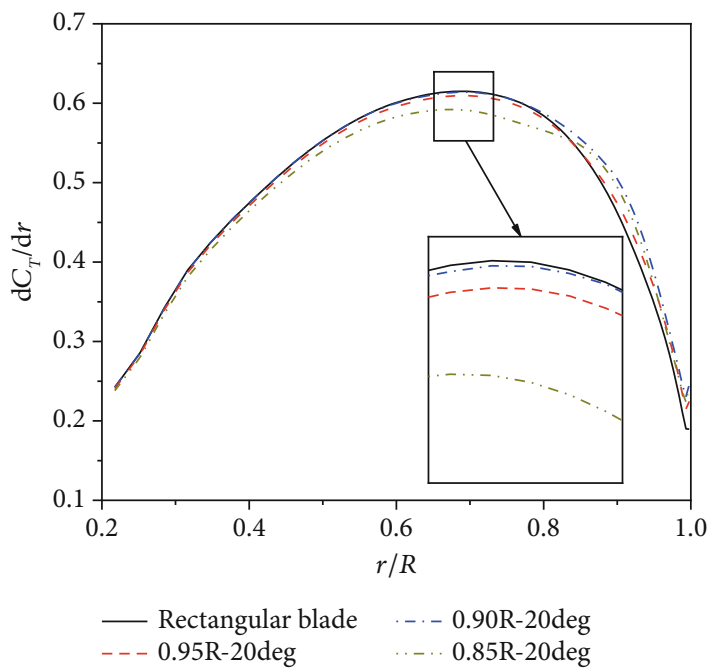

(b) Lower rotor

FIGURE 18: Span thrust distribution $\left(\psi=90^{\circ}, \mu=0.7\right)$.

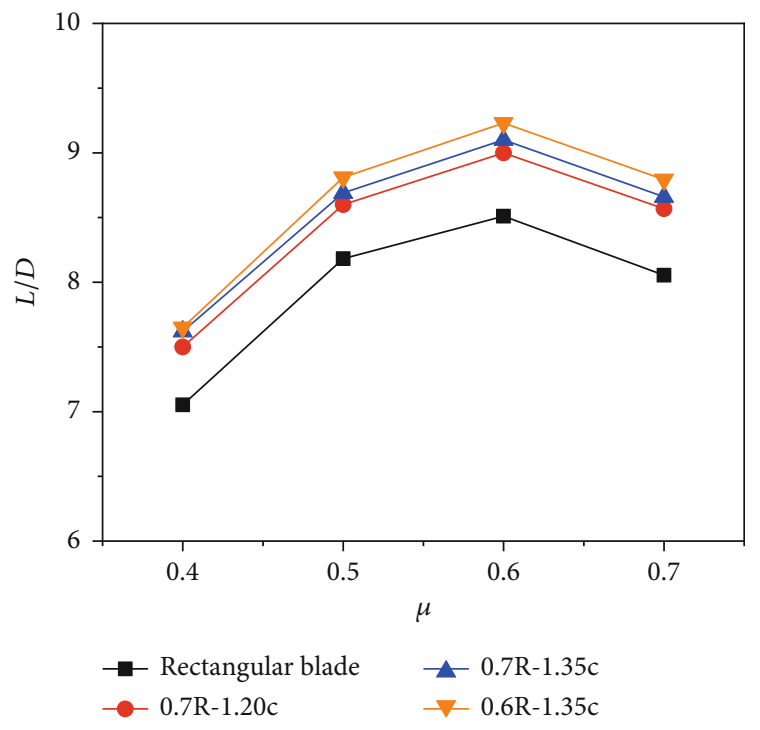

FIGURE 19: Lift-to-drag ratio of rotors with different chord distributions. advancing side. Compared with the rectangular blade, elliptic blades narrow the separation area and could reduce shockinduced drag.

\section{Blade Geometry Design of Coaxial Rotor}

4.1. Optimization Method. Blade geometry optimization is a complex multivariable problem, in which the CFD simulation with trimming of coaxial rotors is time consuming and computationally expensive. Therefore, an optimization method combining the surrogate-based approach and genetic algorithm is implemented in blade geometry shape design in this paper. This method is developed on previous work [25]. Firstly, a surrogate-based approach is established based on LHS (Latin Hypercube Sampling) method and RBF (Radial Basis Function) technique. Then, it is transplanted in the process of genetic algorithm to evaluate the fitness (objective function value).

Figure 23 shows the full optimization flowchart of the coaxial rigid rotor blade geometry. In this flowchart, the surrogate-based approach reduces the calculation amount 


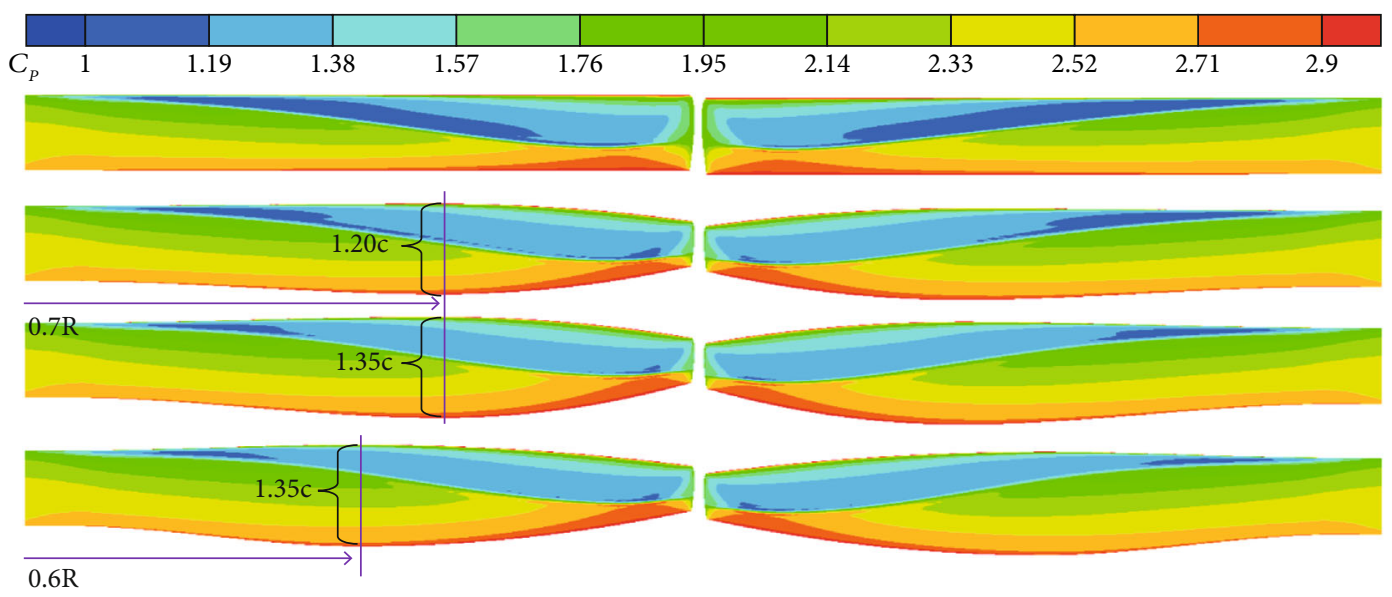

Figure 20: Pressure distribution on the upper surface of the upper $\operatorname{rotor}\left(\psi=90^{\circ}, \mu=0.7\right)$.

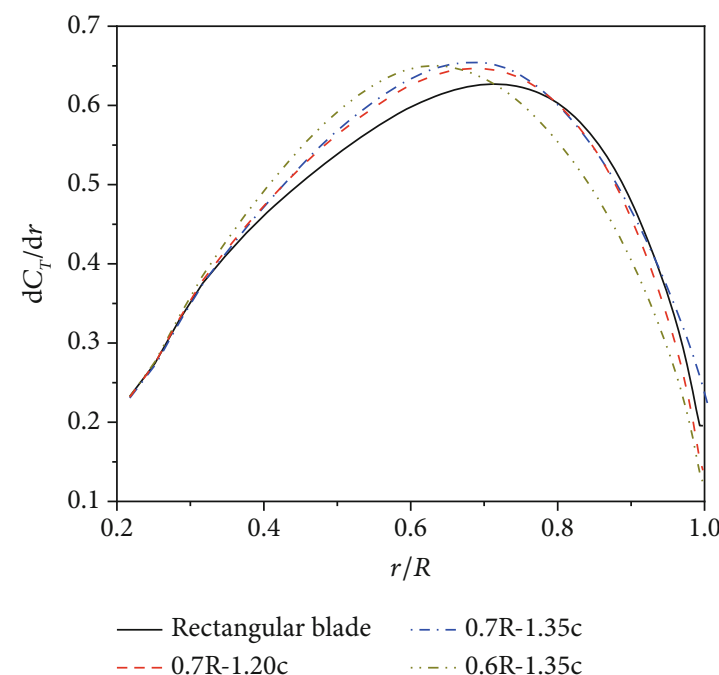

(a) Upper rotor

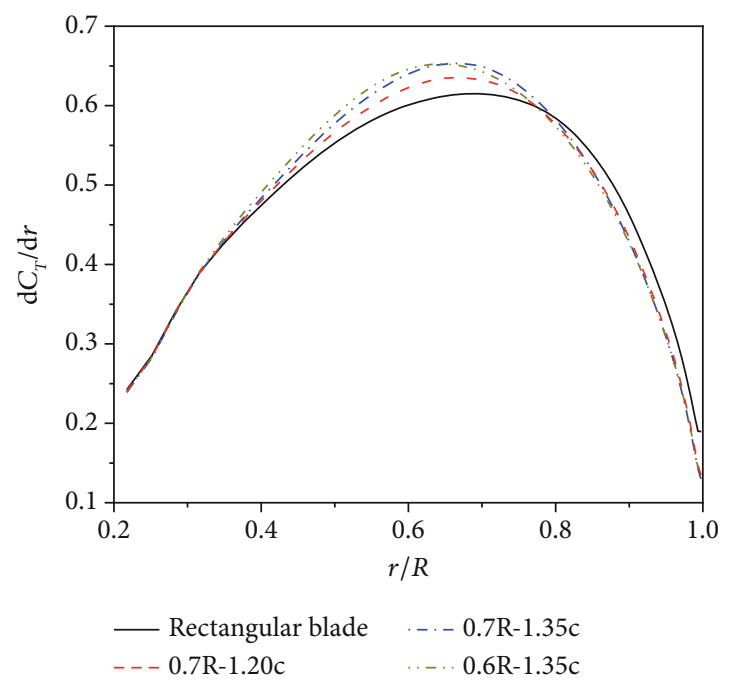

(b) Lower rotor

Figure 21: Span thrust distribution $\left(\psi=90^{\circ}, \mu=0.7\right)$.
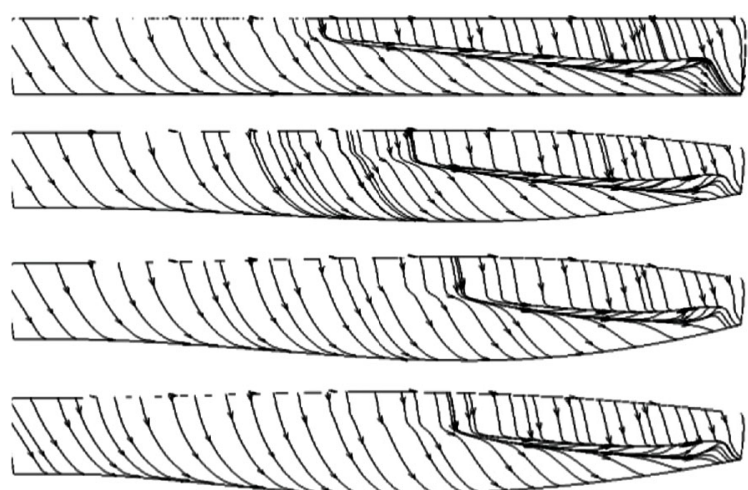

FIGURE 22: Streamline on the upper surface of the upper rotor $\left(\psi=90^{\circ}, \mu=0.7\right)$. in fitness function evaluation. Selected individuals with higher fitness are simulated using CFD solver and then added to the initial population as new parents. The accuracy of the surrogate-based approach is improved with the addition of new parents predicted by high-accuracy CFD solver.

4.2. Parameterization of Blade Planform. Based on the former geometrical parameter analysis, a baseline planform combining elliptic shape feature and swept-back tapered tip is parameterized as shown in Figure 24. This type of planform could concentrate the blade area on the middle part which is in favor of optimizing the airload distribution, and the swept-back tapered tip could reduce the compressibility of the advancing blade tip. There are 8 control points, P1 P8, that define the blade planform. P7 and P8 are fixed at $0.25 \mathrm{R}$ spanwise position. The spline segments between P7 and P1, P8 and P4, and P4 and P5 are defined by cubic functions. 


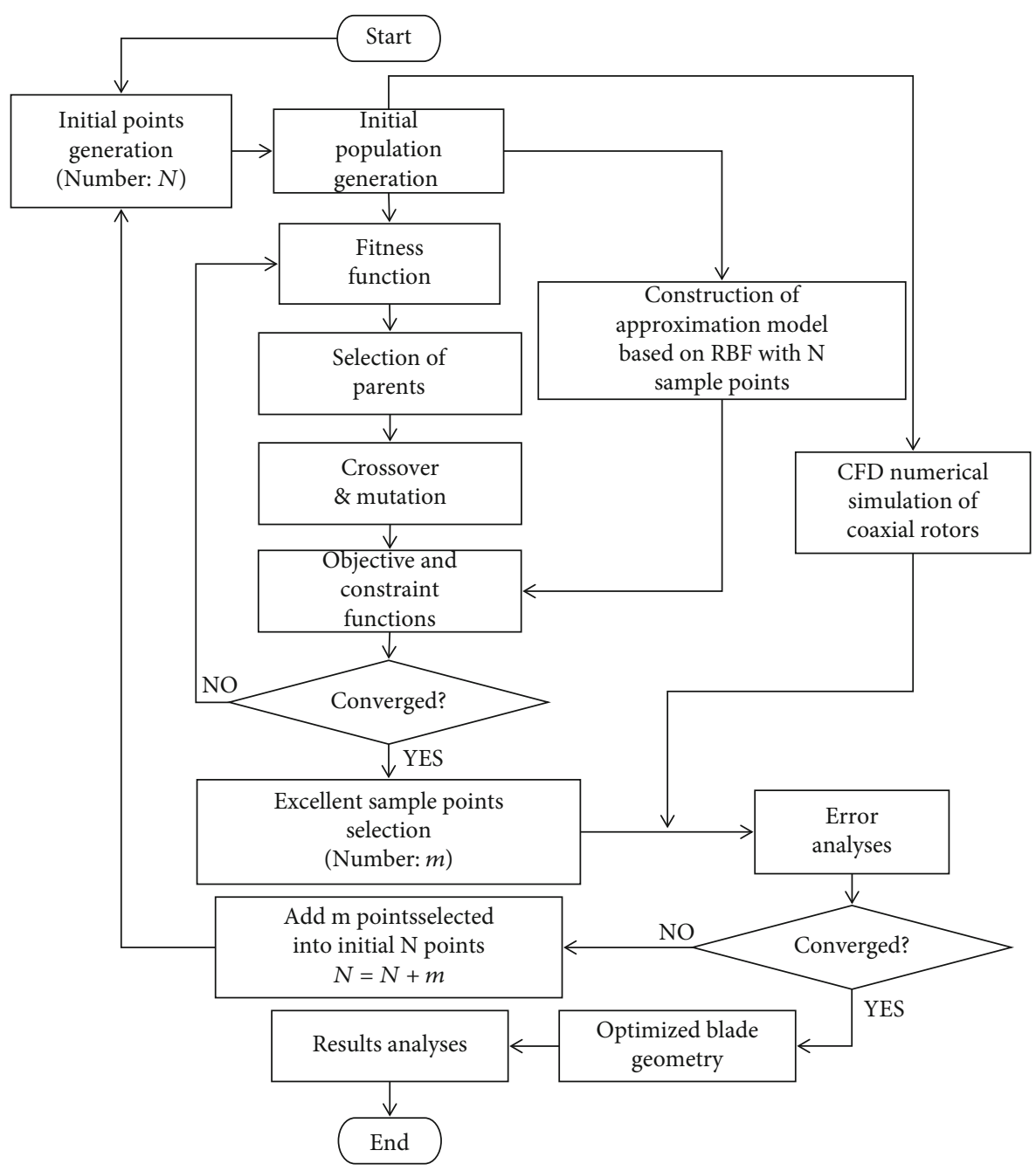

FIGURE 23: Flowchart of coaxial rotor blade geometry optimization procedure.

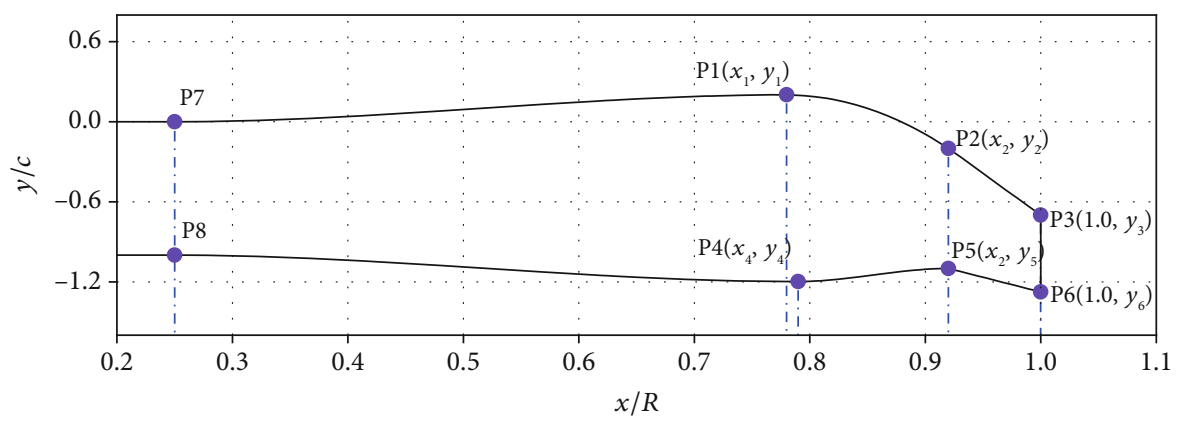

FIGURE 24: Parameterized blade geometry of the coaxial rotor.

The spline segment between $\mathrm{P} 1$ and $\mathrm{P} 2$ is defined by a parabolic function. The spline segments between P3 and P6 and P2 and P3 are defined by linear functions. Except point P5, every connection point is also the tangent point of two segments it connects. For simplification, $x 4$ is set as equal to $x 2$, so there are 8 design variables, $\{x 1, x 2, y 1, y 2, y 3, y 4, y 5, y 6\}$.

Then, the blade geometry is determined by functions as follows:
(1) In the leading edge:

$y=\left\{\begin{array}{l}a_{1}(x-0.25)^{3}+b_{1}(x-0.25)^{2}, \quad 0.25<x \leq x_{1}, \\ k_{1}\left(x-x_{1}\right)^{2}+y_{1}, \quad x_{1}<x \leq x_{2}, \\ k_{2}\left(x-x_{2}\right)+y_{2}, \quad x_{2}<x \leq 1.0\end{array}\right.$ 
TABLE 3: Airfoil distribution.

\begin{tabular}{lcc}
\hline Spanwise position $(/ \mathrm{R})$ & Starting point & End point \\
\hline $0.2 \sim 0.25$ & DBLN526 & OA209 \\
$0.25 \sim\left(x_{1}-0.1\right)$ & OA209 & OA209 \\
$x_{1} \sim x_{2}$ & OA209 & OA206 \\
$x_{2} \sim 1.0$ & OA206 & OA206 \\
\hline
\end{tabular}

where

$$
\left\{\begin{array}{l}
a_{1}=2 \cdot \frac{-y_{1}}{\left(x_{1}-0.25\right)^{3}}, \\
b_{1}=3 \cdot \frac{y_{1}}{\left(x_{1}-0.25\right)^{2}}, \\
k_{1}=\frac{y_{2}-y_{1}}{\left(x_{2}-x_{1}\right)^{2}}, \\
k_{2}=\frac{y_{3}-y_{2}}{1.0-x_{2}} .
\end{array}\right.
$$

(2) In the trailing edge

$y=\left\{\begin{array}{l}a_{2}(x-0.25)^{3}+b_{2}(x-0.25)^{2}-1.0, \quad 0.25<x \leq x_{4}, \\ a_{3}\left(x-x_{2}\right)^{3}+b_{3}\left(x-x_{2}\right)^{2}+y_{5}, \quad x_{4}<x \leq x_{2}, \\ k_{2}\left(x-x_{2}\right)+y_{5}, \quad x_{2}<x \leq 1.0,\end{array}\right.$

where

$$
\left\{\begin{array}{l}
a_{2}=2 \cdot \frac{-\left(y_{4}+1.0\right)}{\left(x_{4}-0.25\right)^{3}}, \\
b_{2}=3 \cdot \frac{\left(y_{4}+1.0\right)}{\left(x_{4}-0.25\right)^{2}}, \\
a_{3}=2 \cdot \frac{-\left(y_{4}-y_{5}\right)}{\left(x_{4}-x_{2}\right)^{3}}, \\
b_{3}=3 \cdot \frac{\left(y_{4}-y_{5}\right)}{\left(x_{4}-x_{2}\right)^{2}}, \\
k_{2}=\frac{y_{6}-y_{5}}{1.0-x_{2}} .
\end{array}\right.
$$

The design variables are constrained as follows to maintain the rotor solidity and geometrical features of the swept-back tapered tip and nonlinear chord distribution:

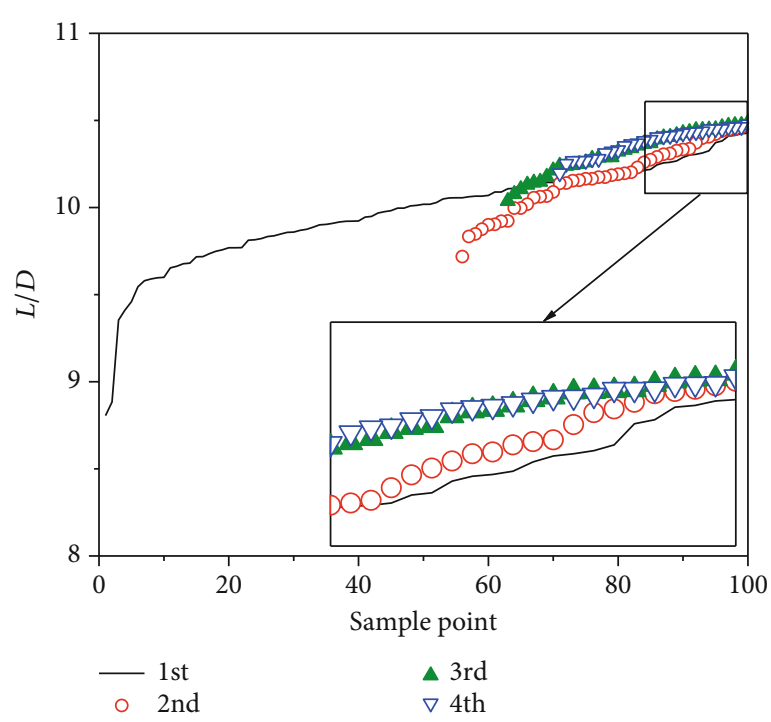

FIgURE 25: Objective value distribution of four generations arranged from lowest to highest.

$$
\begin{cases}0.65<x_{1}<0.7, & 0.1<y_{1}<0.3 \\ -0.8<y_{2}<0.2, & 0.85<x_{2}<0.95 \\ -0.8<y_{3}<y_{2}, & -1.3<y_{4}<y_{1}-1.0 \\ y_{4}<y_{5}, \quad 0.3<y_{3}-y_{6}<y_{2}-y_{5}\end{cases}
$$

Coaxial rigid rotor configuration chosen in the optimization has two 4-bladed rotors, and the rectangular blade is used for comparison of rotor aerodynamic performance. Both rectangular blade and coaxial rotor blade have a diameter of $5.2 \mathrm{~m}$, and the root chord length is $0.2 \mathrm{~m}$. The initial azimuthal angles of four blades of the upper rotor are set at $45^{\circ}, 135^{\circ}, 225^{\circ}$, and $315^{\circ}$. For the lower rotor, they are set at $0^{\circ}, 90^{\circ}, 180^{\circ}$, and $270^{\circ}$. The clearance between two rotors is $0.15 \mathrm{R}$. Airfoil distribution of coaxial rotor is shown in Table 3. There are three kinds of airfoils employed in the blade as shown in the table. Twist distribution has 2 parts, linear twist rate $=14^{\circ} / \mathrm{m}$ inboard (from $0.2 \mathrm{R}$ to $0.4 \mathrm{R}$ ) and $-8^{\circ} / \mathrm{m}$ outboard (from $0.4 \mathrm{R}$ to blade tip). Positive twist rate inboard could reduce the adverse effect caused by reverse-flow region.

The design condition is at $\mu=0.6$ (forward velocity is $210 \mathrm{kts}$ ), and blade-tip Mach number of rotation is 0.528 . Total thrust coefficient is set at 0.013 , and the lift offset is set at 0.35 by the trimming procedure during the optimization. All 8 design variables $\{x 1, x 2, y 1, y 2, y 3, y 4, y 5, y 6\}$ are normalized into $v_{1} \sim v_{8}\left(v_{n} \in[0,1], n=1,2, \cdots 8\right)$. The objective function is the equivalent lift-drag ratio; therefore, it is an optimization problem with eight dimensions and one objective.

$$
\left\{\begin{array}{l}
\text { Design variables : } v_{1}, v_{2}, v_{3}, v_{4}, v_{5}, v_{6}, v_{7}, v_{8}, \\
\text { Objective functions : Maximum }\left(\frac{L}{D}\right)
\end{array}\right.
$$




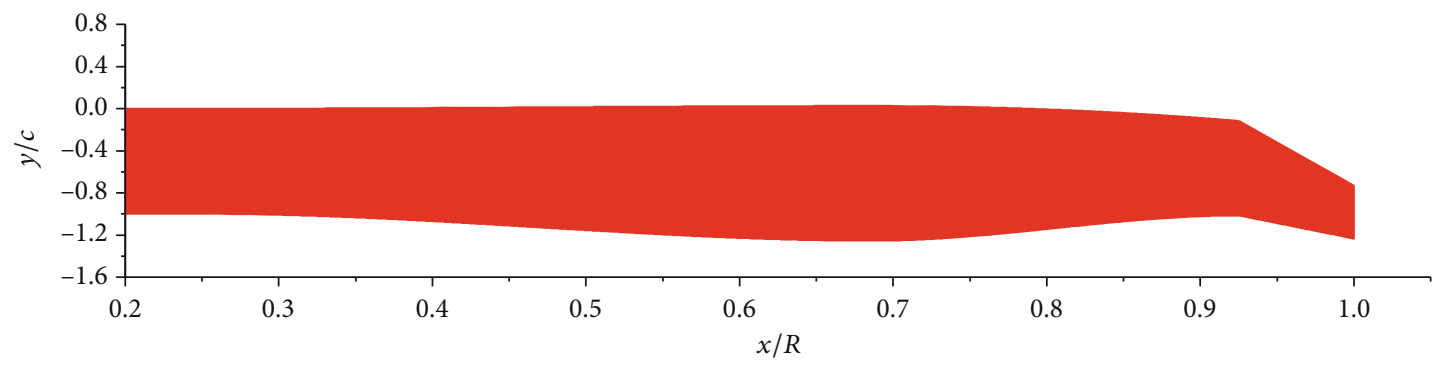

Figure 26: Optimized blade geometry.

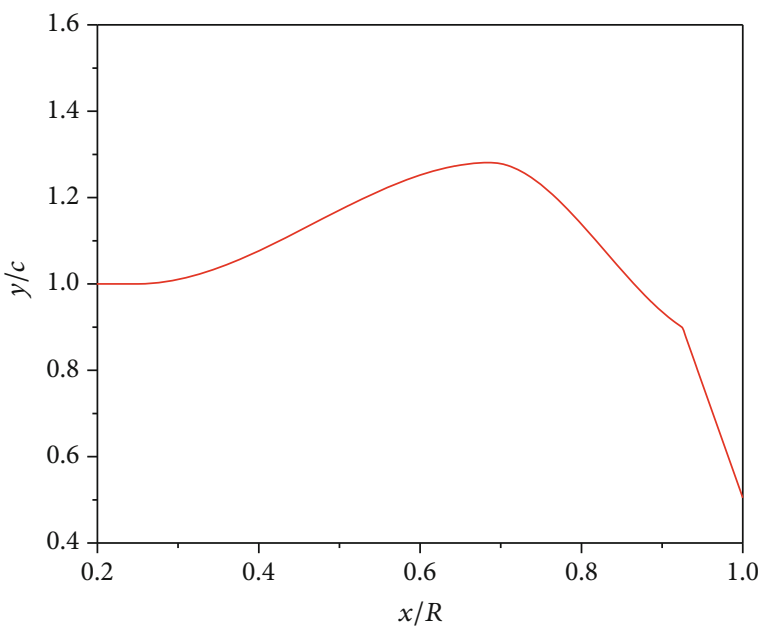

FIGURE 27: Chord distribution of the optimized blade (normalized by the root chord length).

4.3. Results and Analyses. There are 100 sample points in the initial sampling which have 8 normalized variables. After optimization, a converged result is obtained. Figure 25 shows the value of the objective function $L / D$ of each generation that is arranged from the lowest to the highest. The response value of the sample point is higher and higher with the development of optimization, which means the process moves along a favorable direction. In the 4 th generation, the optimization is regarded as converged according to error analyses. The optimized blade shape with a tapered swept-back tip and nonlinear chord distributions is shown in Figure 26, and its chord distribution is shown in Figure 27.

Aerodynamic performance analyses of the optimized rotor are carried out. Figure 28 gives lift-drag ratio of the optimized blade compared with the baseline rectangular blade. Under the current operating condition $(\mu=0.6)$, the lift-drag ratio of the optimized rotor increases about $30 \%$, from 8.06 of the baseline rotor to 10.48. Although the optimization is performed at an advance ratio of 0.6 , the forward flight aerodynamic performance is improved in a wide range of advance ratios. Moreover, the effective-equivalent lift-drag ratio of the coaxial rotor with optimized shape and single NACA0012 airfoil distribution is 9.53. This result manifests the advantage of the optimized planform.

Figure 29 gives the streamline distribution of the upper rotor over the blade surface on the advancing side. Aero-

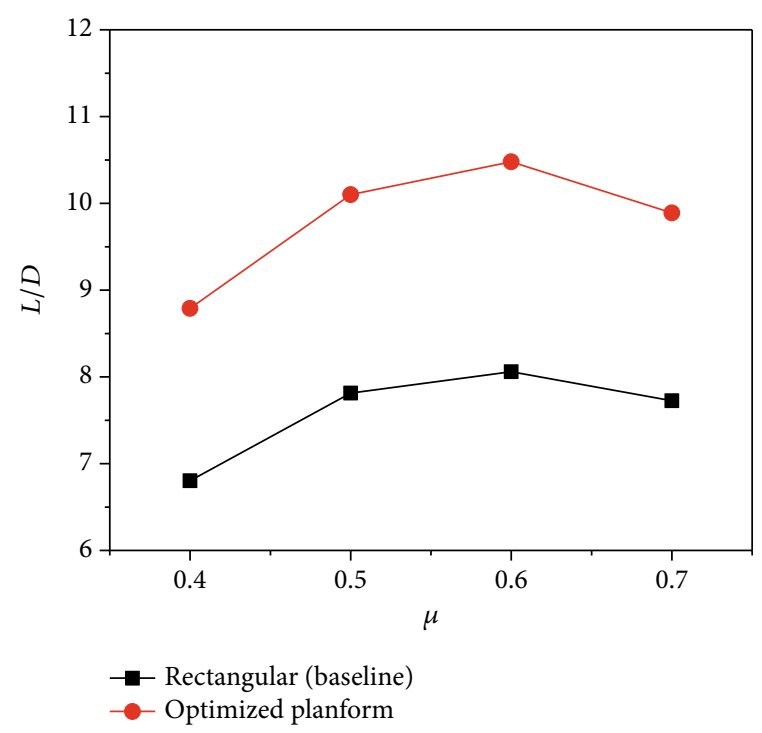

FIgURE 28: The lift-drag ratio of optimized blade and baseline blade.

dynamic characteristics of the upper and lower rotors have little difference at high advance ratios, so that only the numerical simulated results of the upper rotor are presented here. It exhibits that the intense shock wave occurs over the outboard rectangular blade in high-speed forward flight. The shock on the optimized blade is clearly weakened, and the airload distribution along the spanwise of the blade is more reasonable.

Figure 30 shows the blade surface pressure contours in 3 typical azimuthal locations, $0,1 / 16 \mathrm{Rev}$, and $1 / 8 \mathrm{Rev}$. The second location is where the upper rotor meets the lower rotor. There are obvious negative pressure regions on two blades on the advancing side, and the negative pressure region on the surface of the optimized blade has been reduced compared with the baseline. This improvement is mainly due to the reduction of the velocity component normal to the blade leading edge brought by the tapered swept-back distribution. The large area in the middle blade segment provides most of the total lift, and the blade tip is offloaded. This also explains the weakness phenomenon of shock wave in the outboard part of the blade as shown in Figure 29.

Although the noise properties are not considered in the optimization design, the acoustic characteristics of the baseline rotor and optimized rotor are also calculated. Figure 31 


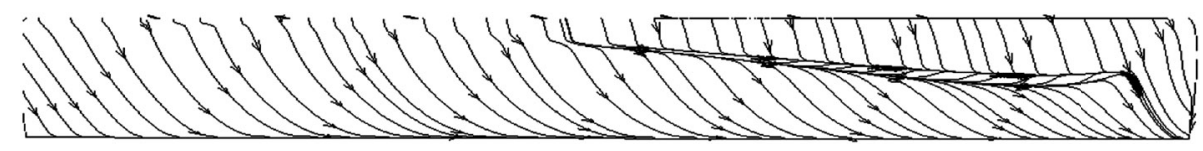

(a) Baseline rotor blade

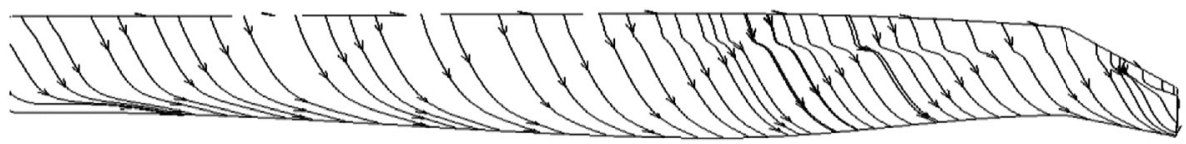

(b) Optimized rotor blade

FiguRE 29: Streamline distributions of the baseline upper rotor and the optimized upper rotor $\left(\psi=90^{\circ}\right)$.

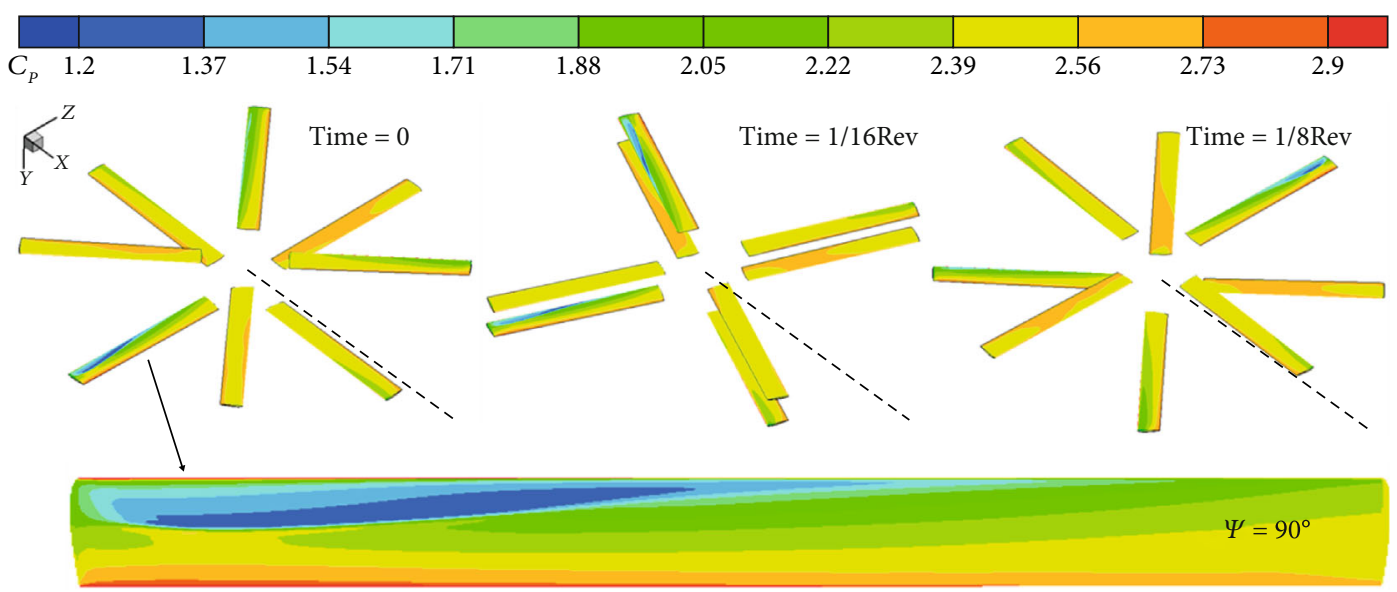

(a) Baseline rotor blade

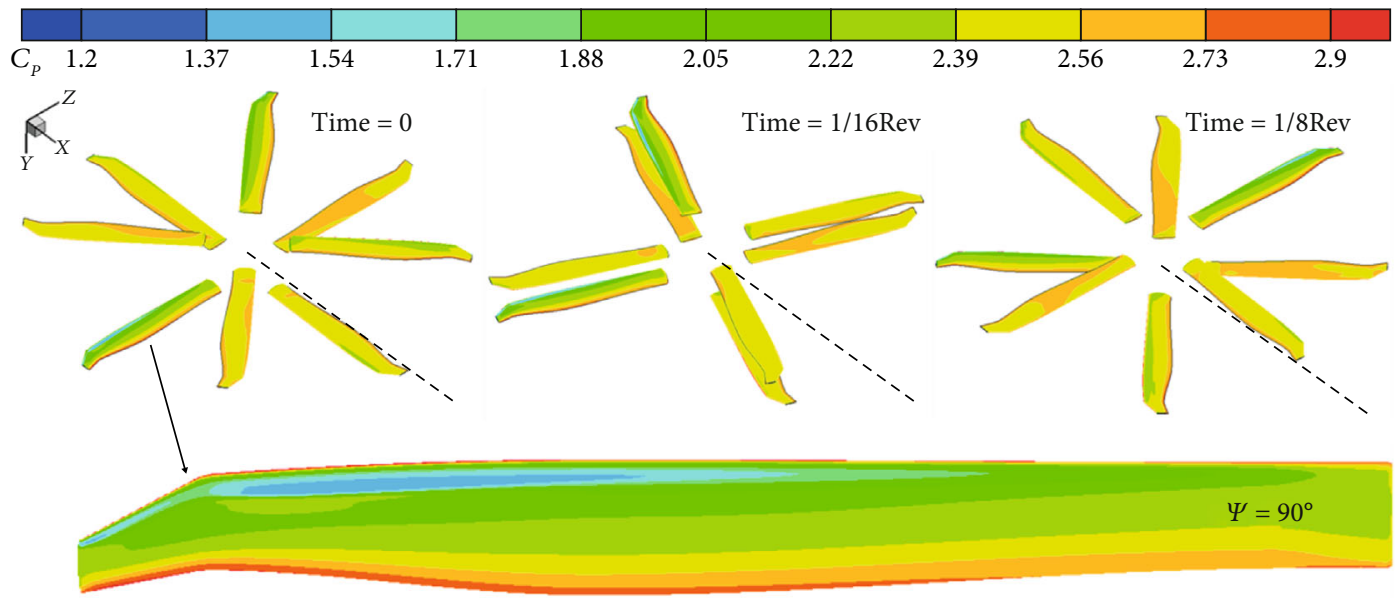

(b) Optimized rotor blade

Figure 30: Blade surface pressure contour distribution of baseline rotor and optimized rotor.

shows the sound pressure history ahead of the rotor plane and noise directivity patterns in the rotor plane. Severe HSI (high-speed impulsive) noise occurs on the coaxial rotor in high-speed forward flight, and the rotor noise has a strong radiation directivity pattern mostly forward the rotor plane. It can be seen from the figure that the value of negative sound pressure peak is decreased about $50 \%$, and the sound pressure level is about $7 \mathrm{~dB}$ lower. Therefore, the optimized blade geometry also contributes to improving noise characteristics.

\section{Conclusions}

A coaxial rotor solver developed on CLORNS was applied to simulate the aerodynamics of the coaxial rotor in forward flight. The flow-field features of the lift offset coaxial rotor at high advance ratio were analyzed. Influences of two geometrical features-swept-back tip and elliptic chord distribution-on rotor performance were studied. Then, a baseline planform combining swept-back tapered tip and nonlinear chord distribution was put forward and used in blade 


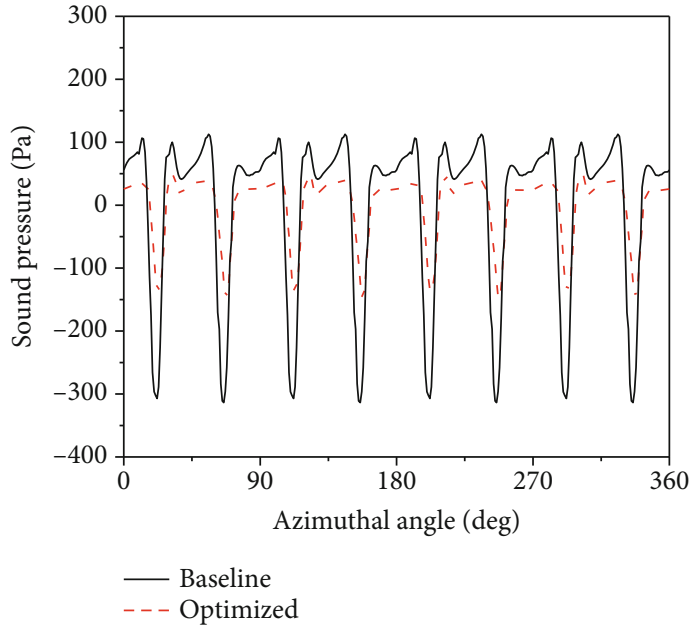

(a) Time history forward the rotor plane

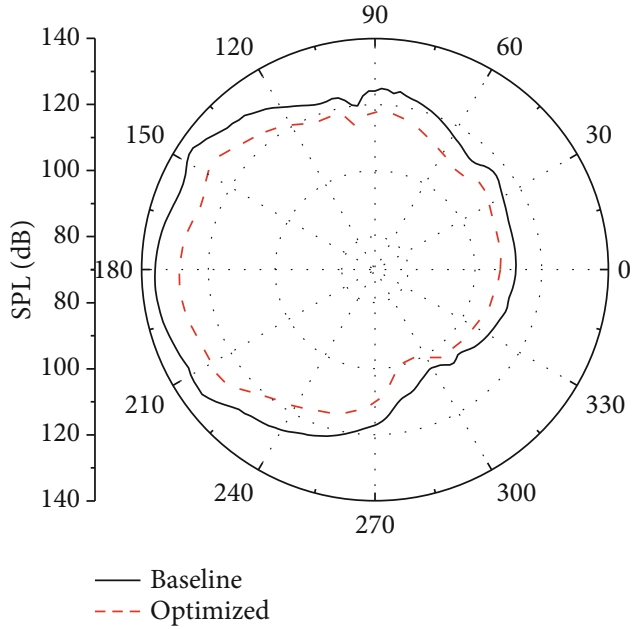

(b) Directivity patterns in the rotor disc

Figure 31: Acoustic characteristics of baseline rectangular blade and optimized blade.

planform optimization. The optimization method combines surrogate-based approach and GA with acceptable computation cost and accuracy. Performance analyses of the optimized coaxial rotor show an obvious improvement on rotor efficiency in forward flight. There are some conclusions that can be drawn from the present researches:

(1) The coaxial rotor solver developed on CLORNS provides reasonable aerodynamic performance predictions. At high advance ratio, the advancing blade tip suffers strong compressibility and the retreating blade root suffers severe reverse flow, which brings adverse effects on rotor efficiency

(2) Swept-back tip mainly influences the aerodynamic characteristics of the blade tip region on the advancing side by reducing the compressibility. Compared with the rectangular shape, the elliptic chord distribution of the blade planform has smaller area in the root and tip, which decreases the drag generated by the blade root in reverse flow and blade tip on the advancing side. Larger area is placed in the middle blade, which benefits a favorable airload distribution

(3) The combination of surrogate-based approach and GA makes the optimization cost less computation than only GA and has a higher accuracy than only surrogatebased approach. The efficiency of the optimized rotor is significantly increased compared with the rectangular blade. The optimized geometry also contributes to improving the noise characteristics, and high-speed impulsive noise in high-speed forward flight is decreased compared with the rectangular rotor

(4) The optimized blade is the optimal solution under current constraints, which is focused on high-speed performance. Hover performance was not involved in the optimization, considering the power provided by the engine is definitely enough to supply hover flights. However, the interferences in the rotor flow- field of hover and low advance ratio are more severe than high-speed forward flights. Further geometry design of coaxial rotor should take hover and lowspeed performance into consideration

The objective of this investigation was to understand the geometrical parameter influences on the forward performance of the coaxial rotor. And the optimization focused on planform design. In the further research, the $2 \mathrm{D}$ airfoil section design will be coupled with the 3D blade geometry design in order to obtain a better coaxial rotor. Airfoil configuration and twist distribution also will be taken into considerations when designing the blade geometry of coaxial rotors.

\section{Nomenclature}

$\begin{array}{ll}c: & \text { Blade chord } \\ C_{D}: & \text { Drag coefficient } \\ C_{P}: & \text { Pressure coefficient } \\ C_{M x}, C_{M z}: & \text { Rolling/pitching moment coefficient } \\ C_{Q}: & \text { Torque coefficient } \\ C_{L}: & \text { Lift coefficient } \\ L / D: & \text { Lift-to-drag ratio } \\ \text { LOS: } & \text { Lift offset (\%) } \\ \text { Ma: } & \text { Mach number } \\ R: & \text { Blade radius } \\ V_{\text {tip }}: & \text { Blade tip speed } \\ \theta: & \text { Pitch angle (deg) } \\ \theta_{0}, \theta_{1 c}, \theta_{1 s}: & \text { Collective/longitudinal cyclic/lateral cyclic pitch } \\ \mu: & \text { angle (deg) } \\ \psi: & \text { Advance ratio } \\ U: & \text { Azimuth angel } \\ L: & \text { Upper rotor } \\ & \text { Lower rotor. }\end{array}$

\section{Data Availability}

The data used to support the findings of this study are available from the corresponding author upon request. 


\section{Conflicts of Interest}

The authors declare that they have no conflicts of interest.

\section{References}

[1] C. P. Coleman, A Survey of Theoretical and Experimental Coaxial Rotor Aerodynamic Research, NASA TP-3675, 1997.

[2] R. D. Harrington, Full-Scale-Tunnel Investigation of the StaticThrust Performance of a Coaxial Helicopter Rotor, NACA TN2318, 1951.

[3] Dingeldein, Wind-Tunnel Studies of the Performance of Multirotor Configurations, NACA TN-3236, 1954.

[4] N. L. Barbely, N. M. Komerath, and L. A. Novak, A Study of Coaxial Rotor Performance and Flow Field Characteristics, Georgia Institute of Technology Atlanta United States, 2016.

[5] A. Bagai and J. G. Leishman, "Free-wake analysis of tandem, tilt-rotor and coaxial rotor configurations," Journal of the American Helicopter Society, vol. 41, no. 3, pp. 196-207, 1996.

[6] R. E. Brown, "Rotor wake modeling for flight dynamic simulation of helicopters," AIAA Journal, vol. 38, no. 1, pp. 57-63, 2000.

[7] R. E. Brown and A. J. Line, "Efficient high-resolution wake modeling using the vorticity transport equation," AIAA Journal, vol. 43, no. 7, pp. 1434-1443, 2005.

[8] H. W. Kim and R. E. Brown, "Computational investigation of small scale coaxial rotor aerodynamics in hover47th AIAA Aerospace Sciences Meeting including The New Horizons Forum and Aerospace Exposition, pp. 9-11, Phoenix, AZ, 2006.

[9] H. W. Kim and R. E. Brown, "Coaxial rotor performance and wake dynamics in steady and maneuvering flight," in American Helicopter Society 62nd Annual Forum, pp. 9-11, Phoenix, AZ, 2006.

[10] J. G. Leishman and S. Ananthan, "Aerodynamic optimization of a coaxial proprotor," Annual Forum Proceedings-American Helicopter Society, , American Helicopter Society, INC, 2006.

[11] J. G. Leishman and S. Ananthan, "An optimum coaxial rotor system for axial flight," Journal of the American Helicopter Society, vol. 53, no. 4, pp. 366-381, 2008.

[12] C. He and J. Zhao, "Modeling rotor wake dynamics with viscous vortex particle method," AIAA Journal, vol. 47, no. 4, pp. 902-915, 2009.

[13] J. Tan, Y. Sun, and G. N. Barakos, "Unsteady loads for coaxial rotors in forward flight computed using a vortex particle method," The Aeronautical Journal, vol. 122, no. 1251, pp. 693-714, 2018.

[14] P. Singh and P. P. Friedmann, "Application of vortex methods to coaxial rotor wake and load calculations in hover," Journal of Aircraft, vol. 55, no. 1, pp. 373-381, 2018.

[15] N. Rajmohan, J. Zhao, and C. He, "A coupled vortex particle/CFD methodology for studying coaxial rotor configurations," in Fifth Decennial AHS Aeromechanics Specialists' Conference, San Francisco, California, 2014.

[16] O. Juhasz, M. Syal, R. Celi et al., "Comparison of three coaxial aerodynamic prediction methods including validation with model test data," Journal of the American Helicopter Society, vol. 59, no. 3, pp. 1-14, 2014.

[17] N. L. Schatzman, Aerodynamics and Aeroacoustic Sources of a Coaxial Rotor, 2018.
[18] R. Singh and H. Kang, "Computational investigations of transient loads and blade deformations on coaxial rotor systems," in Paper AIAA 2015-2884, 33rd American Institute of Aeronautics and Astronautics Applied Aerodynamics Conference, Dallas, TX, 2015.

[19] W. Johnson, A. M. Moodie, and H. Yeo, Design and Performance of Lift-Offset Rotorcraft for Short-Haul Missions, National Aeronautics and Space Administration Moffett Field Ca Ames Research Center, 2012.

[20] H. Yeo and W. Johnson, "Investigation of maximum blade loading capability of lift-offset rotors," Journal of the American Helicopter Society, vol. 59, no. 1, pp. 1-12, 2014.

[21] A. Bagai, "Aerodynamic design of the X2 technology demonstrator $^{\mathrm{TM}}$ main rotor blade," in Proceedings of the 64th Annual Forum of the American Helicopter Society, pp. 29-44, Montreal, Canada, 2008.

[22] Z. Qijun, Z. Guoqing, W. Bo, Q. Wang, Y. Shi, and G. Xu, "Robust Navier-Stokes method for predicting unsteady flowfield and aerodynamic characteristics of helicopter rotor," Chinese Journal of Aeronautics, vol. 31, no. 2, pp. 214-224, 2018.

[23] Z. Zheng, Z. Qijun, and Y. Xin, "Numerical investigations on aerodynamic and acoustic characteristics of a rigid coaxial rotor in hover," in The 6th Asian/Australian Rotorcraft Forum Conference, Kanazawa, Japan, 2018.

[24] Qijun Z, Z. Zheng, Y. Xin, and W. Bo, "Aerodynamic geometry optimization of coaxial rigid rotors in forward flight," in Proceedings of the 74th Annual Forum of the American Helicopter Society, pp. 14-17, Phoenix, Arizona, USA, 2018.

[25] Z. Zhu and Q. Zhao, "Optimization for rotor blade-tip planform with low high-speed impulsive noise characteristics in forward flight," Proceedings of the Institution of Mechanical Engineers, Part G: Journal of Aerospace Engineering, vol. 231, no. 7, pp. 1312-1324, 2016. 